\title{
Evolution of a transcriptional regulator from a transmembrane nucleoporin
}

\author{
Tobias M. Franks, ${ }^{1}$ Chris Benner, ${ }^{1}$ Iñigo Narvaiza, ${ }^{2}$ Maria C.N. Marchetto, ${ }^{2}$ Janet M. Young, ${ }^{3}$ \\ Harmit S. Malik, ${ }^{3,4}$ Fred H. Gage, ${ }^{2,5}$ and Martin W. Hetzer ${ }^{1}$ \\ ${ }^{1}$ Laboratory of Molecular and Cellular Biology, Salk Institute for Biological Studies, La Jolla, California 92037, USA; ${ }^{2}$ Laboratory \\ of Genetics, Salk Institute for Biological Studies, La Jolla, California 92037, USA; ${ }^{3}$ Basic Sciences Division, Fred Hutchinson Cancer \\ Research Center, Seattle, Washington 98109, USA; ${ }^{4}$ Howard Hughes Medical Institute, Fred Hutchinson Cancer Research Center, \\ Seattle, Washington 98109, USA; ${ }^{5}$ Center for Academic Research and Training in Anthropogeny (CARTA), La Jolla, California \\ 92093, USA
}

Nuclear pore complexes (NPCs) emerged as nuclear transport channels in eukaryotic cells $\sim 1.5$ billion years ago. While the primary role of NPCs is to regulate nucleo-cytoplasmic transport, recent research suggests that certain NPC proteins have additionally acquired the role of affecting gene expression at the nuclear periphery and in the nucleoplasm in metazoans. Here we identify a widely expressed variant of the transmembrane nucleoporin (Nup) Pom121 (named sPom121, for "soluble Pom121") that arose by genomic rearrangement before the divergence of hominoids. sPom121 lacks the nuclear membrane-anchoring domain and thus does not localize to the NPC. Instead, sPom121 colocalizes and interacts with nucleoplasmic Nup98, a previously identified transcriptional regulator, at gene promoters to control transcription of its target genes in human cells. Interestingly, sPom121 transcripts appear independently in several mammalian species, suggesting convergent innovation of Nup-mediated transcription regulation during mammalian evolution. Our findings implicate alternate transcription initiation as a mechanism to increase the functional diversity of NPC components.

[Keywords: evolution; hominoid; Pom121; Nup98; transcription; nuclear pore complex (NPC)]

Supplemental material is available for this article.

Received March 15, 2016; revised version accepted April 26, 2016.

The nuclear pore complex (NPC) is an intricate assembly of $~ 30$ different nucleoporins (Nups) that promotes regulated transport of cargo to and from the nucleus (Wente and Rout 2010; Hoelz et al. 2011; Solmaz et al. 2011; Raices and D'Angelo 2012; Hurt and Beck 2015). Although the nucleotide sequences of Nup genes have undergone significant evolutionary changes, the structure of the NPC has remained remarkably well conserved, with essentially no change in protein organization occurring after the appearance of the last eukaryotic common ancestor (LECA) (Devos et al. 2004, 2006; Alber et al. 2007; Brohawn et al. 2008; DeGrasse et al. 2009). In particular, the NPC scaffold Nups, composed of the Nup107/160 and Nup93/205 complexes, typically contain $\alpha$-solenoid and $\beta$-propeller domains that share strong structural similarities to proteins that coat transport vesicles (Devos et al. 2004, 2006; Alber et al. 2007; Brohawn et al. 2008). Based on such similarities, the proto-coatomer hypothesis proposed that NPCs and clathrin, COPI, and COPII vesicle coats share a common evolutionary origin in an early

Corresponding author: hetzer@salk.edu

Article published online ahead of print. Article and publication date are online at http://www.genesdev.org/cgi/doi/10.1101/gad.280941.116. Freely available online through the Genes \& Development Open Access option. membrane-curving module, the "proto-coatomer" (Devos et al. 2004, 2006; Brohawn et al. 2008; Leksa and Schwartz 2010). Other components of the NPC, including the nuclear basket and cytoplasmic filaments, are, for the most part, also well conserved throughout eukaryotes (Field et al. 2014). In contrast, the transmembrane (TM) components of the NPC are often lineage-restricted and have undergone dramatic changes from yeast to humans (Neumann et al. 2010; Field et al. 2014). This is best exemplified by the recent appearance of Pom121, which emerged in vertebrates, where it has an essential role in interphase NPC assembly (Doucet et al. 2010; Dultz and Ellenberg 2010; Funakoshi et al. 2011; Talamas and Hetzer 2011; Field et al. 2014).

Although the composition of the NPC is highly conserved, the functional repertoire of many Nups has expanded throughout evolutionary history. One possible driver of this functional diversity might be the NPC's remarkable ability to act as a scaffold for protein assemblies involved in diverse cellular processes, including the regulation of the cell cycle, DNA damage response, and transcription regulation (Raices and D'Angelo 2012;

(C) 2016 Franks et al. This article, published in Genes \& Development, is available under a Creative Commons License (Attribution 4.0 International), as described at http://creativecommons.org/licenses/by/4.0/. 
Burns and Wente 2014; Ptak et al. 2014; Ibarra and Hetzer 2015). One potential limitation for the NPC in regulating processes such as transcription is the strict localization of Nups to the NPC at the nuclear envelope (NE), which limits the number of genes that can be regulated to those that are in close proximity to the nuclear periphery. For example, in yeast, gene regulation by the NPC is most likely confined to a small subset of genes that are targeted to active transcriptional regions around NPCs to promote gene induction or those that are organized in gene loops, which stimulate rapid transcriptional reinitiation of genes following a period of suppression (Brickner and Walter 2004; Casolari et al. 2004; O'Sullivan et al. 2004; Cabal et al. 2006; Taddei et al. 2006; Tan-Wong et al. 2009; Brickner and Brickner 2011). Thus, in order to increase the number of transcriptional targets that can potentially be regulated by Nups, it would be necessary to uncouple the specific functions of Nups in NPC-mediated transport from those in gene regulation. Consistent with such a notion, previous research in human cells demonstrated that a subset of peripheral Nups can move on and off human NPCs, raising the possibility that Nups' influence on gene expression could extend beyond genomic regions associated with the NE (Rabut et al. 2004). Supporting this hypothesis, the dynamic movement of a subset of Nups, including Nup50, Nup98, and Nup153, was slowed in the presence of transcriptional inhibitory drugs, suggesting a role for Nup98 and potentially other Nups in the regulation of transcription (Griffis et al. 2002, 2004; Buchwalter et al. 2014). A breakthrough in our understanding of the function of mobile Nups came from studies in Drosophila and mammalian cells, which determined that Nup98 and several other peripheral Nups such as Nup50, Nup62, and Nup153 can detach from the NPC, bind to intranuclear promoters distal to the NE, and affect regulation of adjacent genes (Capelson et al. 2010; Kalverda and Fornerod 2010; Liang et al. 2013). Although the mechanism of Nup98-mediated gene regulation in the nucleoplasm is yet to be determined in detail, recent evidence suggests that Nup98 interacts with $C B P / p 300$ and $M B D-R 2 / N S L$ chromatin-modifying complexes in human and Drosophila cells, respectively, suggesting a possible mechanism by which Nup98 promotes an active chromatin state (Kasper et al. 1999; Pascual-Garcia et al. 2014). Most of the evidence collected in Drosophila suggested that only peripheral Nups can exist away from the NPC (Capelson et al. 2010). However, a soluble fraction of the Nup107/160 scaffold complex was recently shown to colocalize with Nup98 in foci in the nucleoplasm of human HeLa cells (Morchoisne-Bolhy et al. 2015).

The finding of bifunctional Nups, which mediate transport at the NPC and transcription in the nucleoplasm, suggests that significant changes might have occurred during evolution to increase functionality without disrupting an existing essential function. Nevertheless, these two functions suggest the possibility of pleiotropy, in which optimal functionality for both transport and transcription functions cannot be accommodated within a single protein-coding gene (Guillaume and Otto 2012), reducing the adaptive capacity of the NPC (Orr 2000). Gene duplication has been proposed as one mechanism that can relieve the deleterious effects of such antagonistic pleiotropy of functions under certain conditions (Guillaume and Otto 2012). However, the possibility of such subfunctionalization in the NPC has not been previously explored.

Here, we identify a variant of the TM Nup gene POM121 that produces a soluble (i.e., non-membranebound) form of Pom121 (sPom121) that lacks its TM domain and is no longer incorporated into the NPC. In hominoids, the sPom121 transcript is expressed from an alternative transcriptional start site that arose from a genomic rearrangement. This novel isoform includes new 5 ' untranslated region (UTR) exons and bypasses the canonical TM-coding exon to encode an $\mathrm{N}$-terminally truncated form of Pom121. Functionally, sPom121 and Nup98 cobind specific gene promoters to regulate transcription in human cells. Thus, sPom121 represents the first validated example of an NPC component that has eschewed its role in nucleo-cytoplasmic transport to specialize in an unrelated process; namely, gene regulation. In addition, we show that SPom121 can promote retention of Nup107/ 160 complexes in the nucleoplasm during NPC formation, suggesting that the evolution of sPom121 brought about dramatic functional expansion of other scaffold Nups in hominoid cells.

\section{Results}

\section{Alternative transcription initiation produces a sPom121 isoform in humans}

While investigating expressed sequence tags (ESTs) for human Pom121 transcripts, we noticed, consistent with previous reports (Funakoshi et al. 2007), that there is an abundance of sequences that contain a noncanonical 5' UTR sequence and lack the TM-coding sequence of Pom121 (Fig. 1A, "sPom121 isoform"). These noncanonical transcripts, here called the sPom121 mRNA, are predicted to initiate at an alternative transcription start site $\sim 40 \mathrm{~kb}$ upstream of the TM-encoding canonical first exon (here called exon 4), include three or four exons in this upstream region, and splice past the exon 4 to the second "canonical" coding exon of Pom121 (exon 5) (Fig. 1A). As a result, we predict that $S P$ Pom 121 transcripts would be translated beginning at an ATG codon in exon 5, encoding an N-terminally truncated form of Pom 121 that is missing the $\mathrm{N}$-terminal TM domain.

To explore the expression of the sPom121 mRNA in human cells and confirm the sPom121 gene model, we conducted 5' RACE (rapid amplification of cDNA ends) experiments in six cell lines: HeLa-C, HeLa, U2OS, IMR90, RPE-1, and SISA. Importantly, a reverse oligo was chosen that anneals downstream from the Pom121 TM-coding region at a site that is predicted to be shared between Pom121 and sPom121 transcripts. The amplified DNA products migrate as a smear at $\sim 1500$ base pairs (bp) (Supplemental Fig. S1A). Cloning and sequencing revealed that most Pom121 5' sequences (22 of $24 \mathrm{HeLa}-\mathrm{C}$ clones, 
A

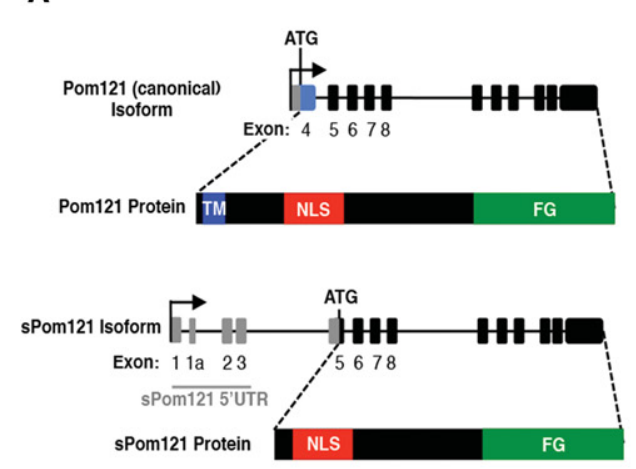

E

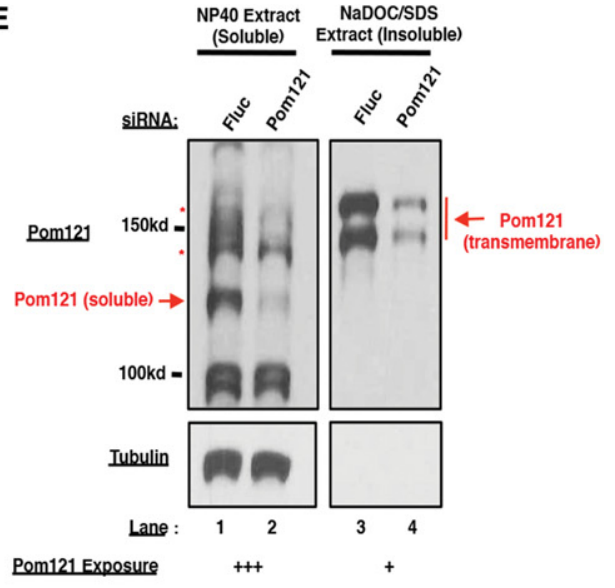

B

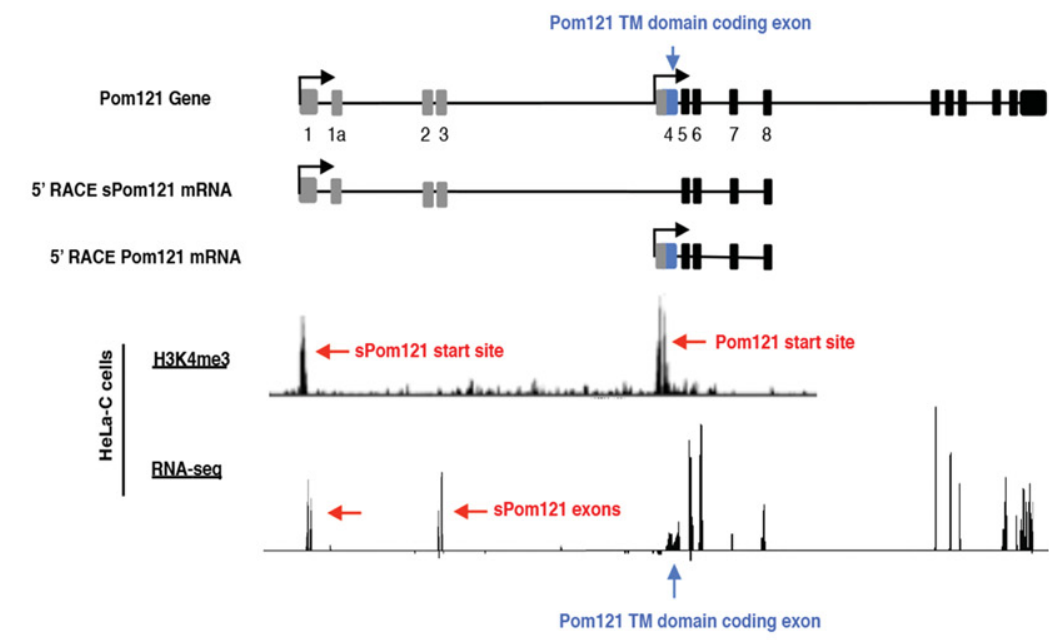

D

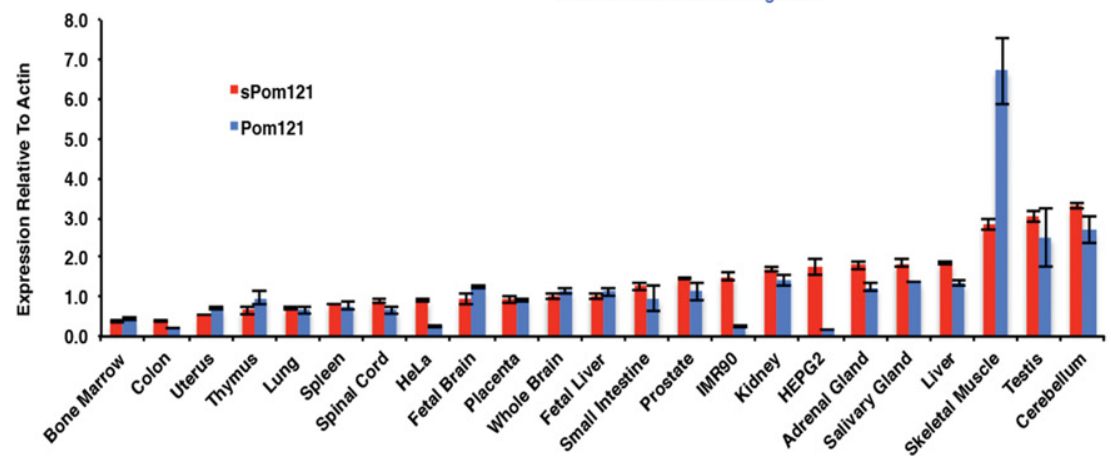

Figure 1. Detection of sPom121 mRNA and protein expression in human cells. (A) Schematic of two putative Pom121 isoforms expressed in humans and confirmed by 5' rapid amplification of cDNA ends (RACE). (Top) Gray boxes indicate 5' UTR-encoding exons, blue boxes indicate TM domain-encoding exon, and black boxes indicate Pom121-encoding exons. (Bottom) The TM domain, nuclear localization signal (NLS), and phenylalanine/glycine (FG) domain are indicated (blue, red, and green, respectively). (B) Schematic showing the annotated $5^{\prime}$ end of the Pom121 gene (shown at the top, "Pom121 gene") compared with that of sPom121 ("5' RACE sPom121 mRNA") and Pom121 ("5' RACE Pom121 mRNA"), identified here by 5' RACE. (C) Histone H3 Lys4 trimethylation (H3K4me3) (top) and RNA sequencing (RNA-seq) (bottom) results from HeLa-C cells. Red arrows are used to indicate active transcriptional start sites (top), sPom121-specific exons (bottom left), or the TM-coding exon of Pom121 (bottom right). (D) sPom121 and Pom121 expression in various tissues. Quantitative PCR (qPCR) analysis of sPom121 (red bars) and Pom121 (blue bars) mRNA levels in multiple tissue types relative to actin. Results are plotted such that the tissue with the lowest sPom121 mRNA expression is at the left, while the tissue expressing the highest levels of sPom121 is shown at the right. Different primers were used to analyze sPom121 and Pom121 cDNA levels, and thus a comparison of sPom121 and Pom121 levels in each tissue cannot be made from these data. (E) Western blot to detect sPom121. Soluble $($ lanes 1,2) and insoluble (lanes 3,4) lysates were electrophoresed and Western blotted, and proteins were detected with a Pom121 antibody (top panels) or tubulin (bottom panels). (Lanes 2,4) Samples that had been treated with Pom121 siRNA are included to identify which bands correspond to Pom121 protein. Pom121 blots were exposed for $30 \mathrm{sec}$ (left blot) or $10 \mathrm{sec}$ (right blot). 
four of five U2OS clones, and four of four IMR90 clones) corresponded to the predicted sPom 121 transcript, which lacks a TM-coding region (Fig. 1B; Supplemental Fig. S1B,D). RACE analysis further revealed that this transcript contains three or four additional exons unique to the sPom121 mRNA (Fig. 1B). In contrast, the other Pom121 transcripts (three of 33 clones for HeLa, U2OS, and IMR90 combined) corresponded to the canonical Pom121 mRNA, which has a short 5' UTR and includes the Pom121 TM domain (Fig. 1B; Supplemental Fig. S1C,D). These results demonstrated that sPom121 and Pom121 mRNAs are the products of two different transcriptional start sites.

To further confirm alternate transcriptional initiation, we conducted chromatin immunoprecipitation (ChIP) experiments in HeLa-C cells with antibodies raised against histone $\mathrm{H} 3$ Lys4 trimethylation (H3K4me3), a histone mark that is a reliable indicator of active promoters, followed by ChIP sequencing (ChIP-seq) experiments. As shown in Figure 1C, H3K4me3 peaks are clearly observed at both the previously identified Pom121 transcriptional start site and an additional upstream start site that corresponds to the $5^{\prime}$ end of the sPom121 RACE product (identified by red arrows). RNA sequencing (RNA-seq) analyses in HeLa-C cells further confirmed the gene model for sPom121, including the newly identified first three exons (Fig. 1C, red leftfacing arrows). In addition to the sPom121-unique exons, reads corresponding to exon 4 (Fig. 1C, blue up-facing arrow) can be used to unambiguously analyze the expression of the canonical Pom121 mRNA. The unique exons of sPom121 were a useful tool to allow us to determine whether sPom121 expression is consistent across all cell types or is tissue-specific. To this end, we synthesized cDNA and conducted quantitative PCR (qPCR) to quantify sPom121 and Pom121 expression levels from a panel of 20 different human tissues. We found that expression of both sPom121 and Pom121 mRNA is widely expressed across tissues, although at varying levels; the highest SPom121 expression is observed in the cerebellum, testis, skeletal muscle, and liver (Fig. 1D; Supplemental Fig. S1E,F).

We next tested whether the sPom121 mRNA produces a functional protein. Using Western blotting, we attempted to unequivocally determine the presence of a soluble, TM-lacking form of Pom121. We first sequentially extracted soluble and then membrane-bound proteins from cells that had been treated with either a control siRNA targeting firefly luciferase (Fluc) or an siRNA that targets a sequence that is shared between Pom121 and SPom121. The lysates were separated by SDS-PAGE and analyzed using specific anti-Pom121 antibodies. We detected a band of $\sim 125 \mathrm{kDa}$ (Fig. 1E, lane 1, red arrow) in the soluble lysate, which correlates with the expected size of a form of Pom121 lacking its N-terminal TM domain. Importantly, the intensity of the $125-\mathrm{kDa}$ band is strongly reduced upon knockdown of Pom121 with an siRNA, suggesting that this protein corresponds to endogenous sPom 121. As expected, full-length Pom121 (Fig. 1E, lanes 3,4) was observed primarily in the insoluble lysate running as a doublet at the expected size of $\sim 150 \mathrm{kDa}$, as would be consistent with a TM domain-containing protein. The ability to extract Pom121 and sPom121 using different conditions further suggests that the two proteins are in distinct complexes.

\section{sPom121 localizes to the nucleoplasm instead of the NPC}

In order to test whether Pom121 and sPom121 have distinct subcellular localization, we conducted immunofluorescence (IF) assays in HeLa-C cells with antibodies raised against three different regions of Pom121. We specifically used HeLa-C cells because they are characterized by intranuclear Nup foci that facilitate visualization of intranuclear Nups by IF (Xu and Powers 2010; MorchoisneBolhy et al. 2015). As expected, we observed Pom121 localization to the nuclear periphery, consistent with localization to the NPC (Fig. 2A,B). In addition to this nuclear rim localization, we observed a pool of intranuclear Pom121 colocalizing with Nup98 in the nucleoplasm (Fig. 2B). This intranuclear localization of sPom121 was confirmed with two additional antibodies that recognize distinct epitopes of the Pom121 protein, suggesting specificity of the signal for the endogenous protein (Fig. 2B; Supplemental Fig. S2A).

To test whether other Nups are recruited to sPom121 foci, we compared its localization with that of other Nups, including components of the nuclear basket, cytoplasmic filaments, and scaffold structure (Fig. 2A). We readily observed components of the Nup107/160 scaffold complex, including Nup96, Nup133, Nup107, and Elys, colocalizing with Nup98 (Supplemental Fig. S2B, panels $2,5,8.11$ ) in the nucleoplasm in $>90 \%$ of cells, similar to what has been shown previously (Morchoisne-Bolhy et al. 2015). In contrast to Pom121 and the Nup107/160 complex, we did not observe nuclear basket components, components of the cytoplasmic filaments, the Nup93/205 scaffold complex, or any of the basket and cytoplasmic filament Nups detected by antibody mAB414 colocalizing with intranuclear Nup98 (Supplemental Fig. S2C). These findings suggest that sPom121-containing structures do not resemble intranuclear NPCs.

Next, we wondered whether SPom121 protein is present in other cell types. To test this, we overexpressed GFPNup98 to induce macroscopic Nup98 foci, which facilitate the detection of potential sPom121. We then probed cells with anti-Pom121 antibodies. Indeed, overexpression of GFP-Nup98 in HeLa, U2OS, and IMR90 cells induces colocalization of Pom121 and GFP-Nup98 in 94\%, $94 \%$, and $86 \%$ of cells, respectively (Supplemental Fig. S2D). In addition, the Nup107/160 complex, but not factors detected by mAB414, was also observed to colocalize with intranuclear GFP-Nup98 in U2OS cells (data not shown).

We considered whether sPom121 foci in the nucleoplasm could be the result of NE invaginations, which can be observed at relatively low frequency in many cell types. We overexpressed full-length rat Pom121-GFP (which contains a TM domain) to test for colocalization 
A
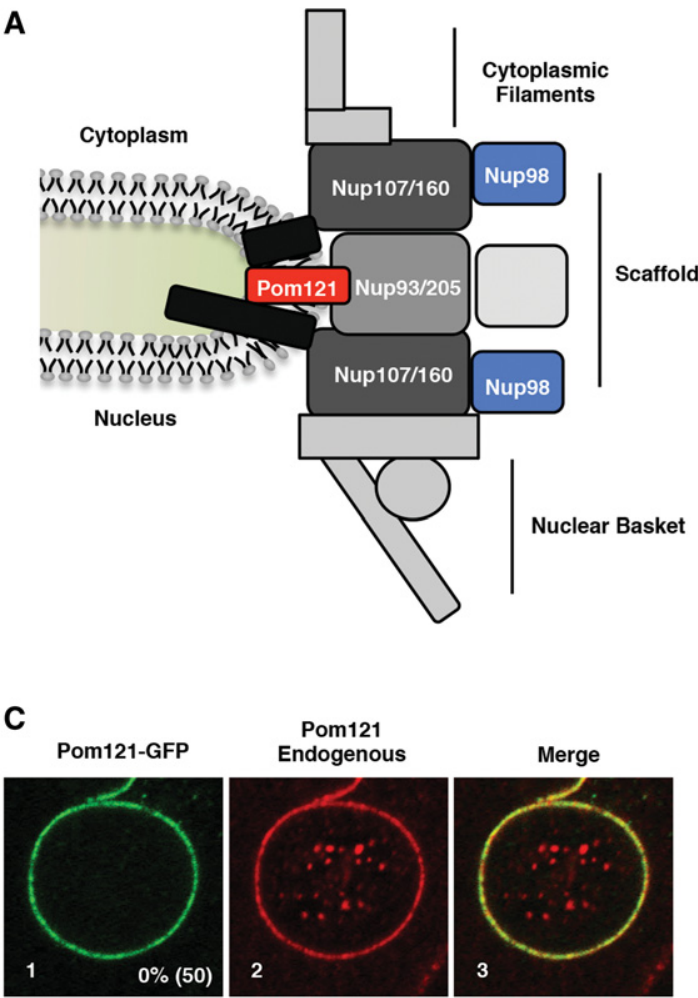

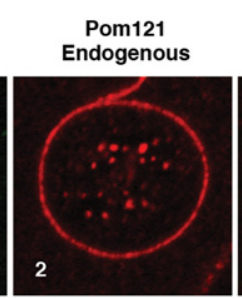

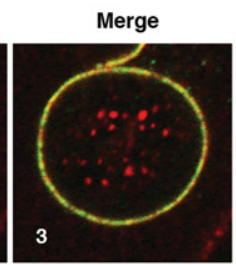

B

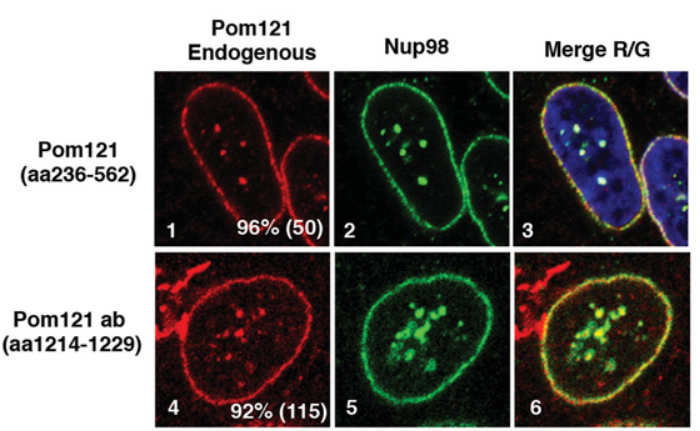

D

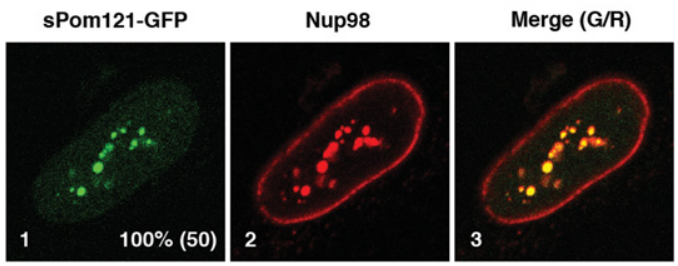

Figure 2. sPom 121 protein localizes in the nucleoplasm of human cells. (A) Schematic of the NPC components. (Gray, nuclear side of the NE) Cytoplasmic filaments; (gray, cytoplasmic side of the NE) nuclear basket; (black) Nup107/160 complex; (dark gray) Nup93-205 complex; (black with Pom121 highlighted in red) TM Nups; (blue) Nup98. (B) IF assays showing localization of endogenous Pom121 (panels 1,4) and Nup98 (panels 2,5) in HeLa-C cells. Merged images are shown in panels 3 and 6. (C) Comparison of localization of transfected rat Pom121-3GFP (panel 1) with endogenous Pom121 (panel 2). A merged image is shown in panel 3. The percentage of cells with intranuclear localization of the Pom121-3GFP is indicated in the bottom right corner of panel 1, while the number of cells quantified is in parentheses. $(D)$ Comparison of sPom121-GFP (rat Pom121 amino acids 241-1200) with Nup98 in HeLa-C cells. The percentage of cells with intranuclear localization of the Pom121-3GFP is indicated in the bottom right corner of panel 1, while the number of cells quantified is in parenthesis.

with endogenous Pom121 in the nuclear interior in HeLaC cells. As expected, Pom121-GFP was observed only at the nuclear rim and not colocalizing with endogenous Pom121 in the nucleoplasm (Fig. 2C). Moreover, we did not observe colocalization of intranuclear Pom121 foci with all NPC components (see above) or with lamina proteins (data not shown), suggesting that sPom121 is not associated with NPCs. These results suggest that endogenous sPom121 likely represents a soluble variant that lacks the N-terminal TM domain of Pom121. To confirm this, we cloned and expressed the GFP-tagged sPom 121 (Pom121 amino acids 241-1200) (Fig. 2D) and conducted IF assays to compare sPom121-GFP localization with that of Nup98. We found that sPom121-GFP (Fig. 2D, panel 1) colocalizes with Nup98 (Fig. 2D, panel 2) and phenocopied the localization of the endogenous SPom121 protein (Fig. 2, cf. D [panel 1] and B [panels 1,4]). Finally, our Western blot analyses (Fig. 1D) confirm that Pom121 and sPom121 are in distinct complexes, with the latter representing a soluble variant (Fig. 1D). We therefore conclude that sPom121 colocalizes in the nucleoplasm with Nup98 and the Nup107/160 complex in a broad range of human cell types.
The nuclear localization signal (NLS) domain of sPom121 is required for colocalization with Nup98

To further understand how sPom121 functions in the nucleoplasm, we wanted to determine how sPom121 colocalizes with Nup98 and the Nup107/160 complex in the nucleoplasm. Previous studies suggest that the NLS domain of Pom121 is crucial for interactions with various Nups at the NPC, including Nup98 and the Nup107/ 160 complex (Mitchell et al. 2010; Yavuz et al. 2010; Shaulov et al. 2011). In addition, the NLS domain is required for most of Pom121's described roles at the NPC (Yavuz et al. 2010; Shaulov et al. 2011). We therefore hypothesized that, like canonical Pom121 at the NPC, sPom121 uses its NLS domain to interact with Nup98 and the Nup107/160 complex except it does so in the nucleoplasm.

To determine which domain of sPom121 is required for colocalization with Nup98, we took advantage of our sPom121-GFP construct (Fig. 2D) and created a series of sPom121 deletion mutants lacking the C-terminal phenylalanine/glycine (FG) domain, the central domain, and portions of the N-terminal NLS domain (Fig. 3A). 
A

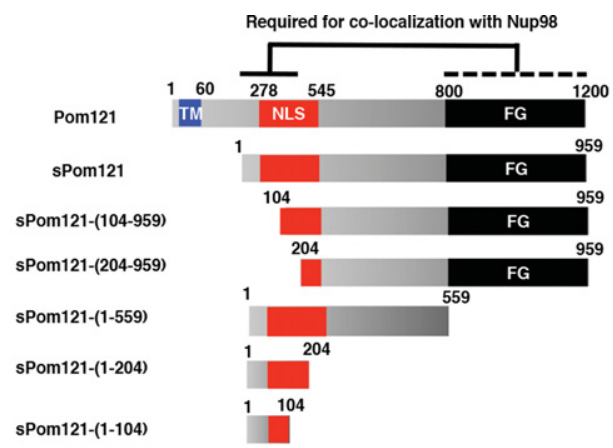

B

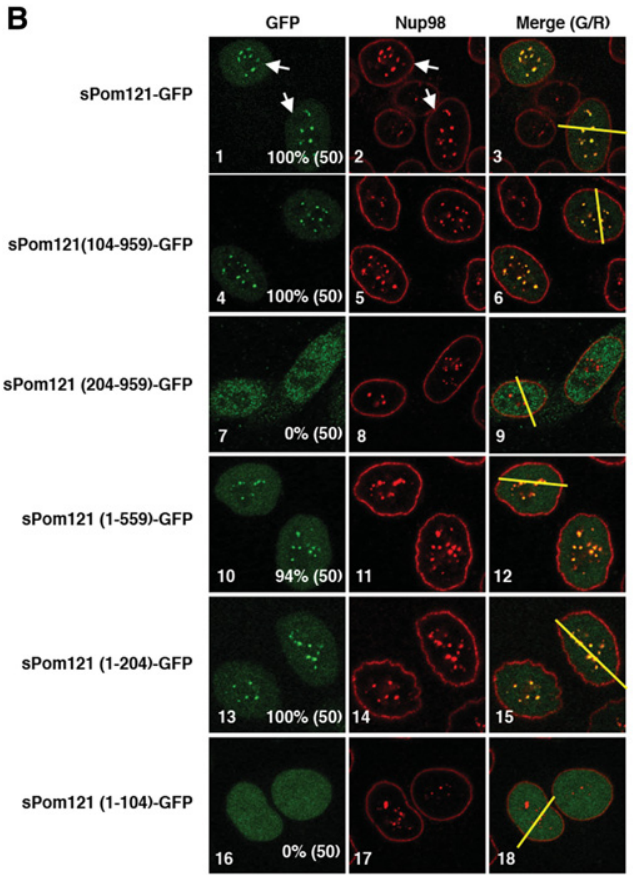

Co-localizes with Nup98 in the nucleoplasm?

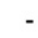

$+++$

$+++$

$-$

$++$

$++$

$-$
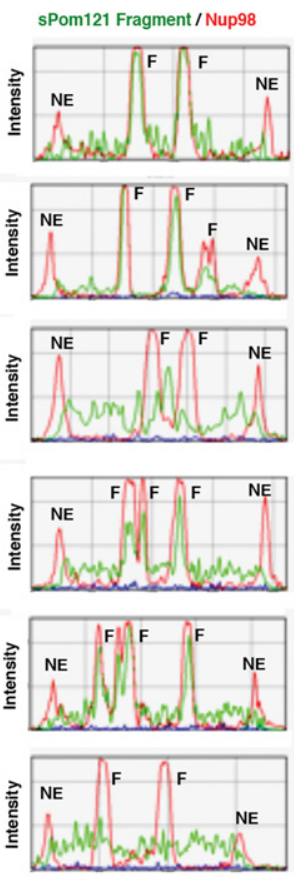

Figure 3. The NLS domain of sPom 121 is required for colocalization with Nup98. $(A)$ Schematic of Pom 121 mutants used to identify the domain required to localize sPom 121 to Nup98 foci. All mutants were cloned from a rat Pom121-3GFP construct that was described previously (Doucet et al. 2010; Talamas and Hetzer 2011). The Pom 121 TM domain (blue), NLS domain (red), and FG domain (black) are shown. The fragment of sPom 121 required for colocalization with Nup98 is indicated with a black bar (top), while the region that has a minor effect on sPom121 localization is indicated with a dotted black bar (top) (B) IF assays showing localization of the sPom 121 mutants listed at the left (panels 1,4,7,10,13,16) and Nup98 (panels 2,5,8,11,14,17). Merged images are shown at the right (in panels $3,6,9,12,15,18$ ). The percentage of cells with the Pom $121 \mathrm{mu}$ tant colocalizing with GFP-Nup98 in the nucleoplasm is shown in the bottom right corner of the left panels, while the number of cells counted is shown in parentheses. (Panels $3,6,9,12,15,18)$ The fluorescence intensity of sPom121 and Nup98 at either the nuclear membrane or nucleoplasmic foci was observed by quantifying the intensity of the yellow lines drawn through a cell cross-section. The intensity graphs are shown at the right. $(\mathrm{F})$ Focus. sPom121 only colocalizes with Nup98 in nucleoplasmic foci but never at the NE.
We assayed both the full-length and deletion sPom121GFP proteins for colocalization with Nup98 using IF analysis. As shown in Figure 3B, amino acids 1-204 of sPom121 are necessary and sufficient for sPom121 colocalization with Nup98 (cf. panels 13 and 14 and the line graph at the right) in the nucleoplasm, while the FG domain appears to serve a minor role, as demonstrated by an increase in diffuse nuclear localization of sPom121 in FG domain mutants (cf. panels 10,11 and 1,2 and see the line graphs at the right). We also observed a marked increase in the size and intensity of Nup98 foci in cells expressing sPom121-GFP (Fig. 3B, panel 2, white arrows), suggesting that sPom121 overexpression can promote the retention of Nup98 and potentially other Nups such as the Nup107/160 complex in intranuclear bodies. We conclude that the NLS domain of sPom121 is required for colocalization with Nup98 and the Nup107/160 complex, while the FG domain of sPom121 plays a secondary role in enhancing the affinity of sPom121 for Nup98 complexes.
sPom121 and Nup98 have similar intranuclear dynamics

The observed colocalization of sPom121 and Nup98 and the previously defined Pom121-Nup98 interaction (Mitchell et al. 2010) suggested that both proteins might function together in the nucleoplasm of human cells to carry out an unidentified function. As a first step to test whether the function of sPom121 in the nucleoplasm is related to that of Nup98, we expressed either GFPNup98 or sPom121-GFP in HeLa-C cells and conducted fluorescence resonance after photobleaching (FRAP) experiments. If both proteins are present in the same complex, we expected to observe similar recovery kinetics of intranuclear sPom121 and Nup98. As shown in Figure 4A, after a short bleach, GFP-Nup98 fluorescence recovery was identical to that of sPom121-GFP (cf. panels 2,3 and 5,6; also, Fig. 4B, cf. the blue trace and the red trace). We wondered whether all Nups that colocalize with Nup98 share the same kinetics or whether Nup98 and 
A

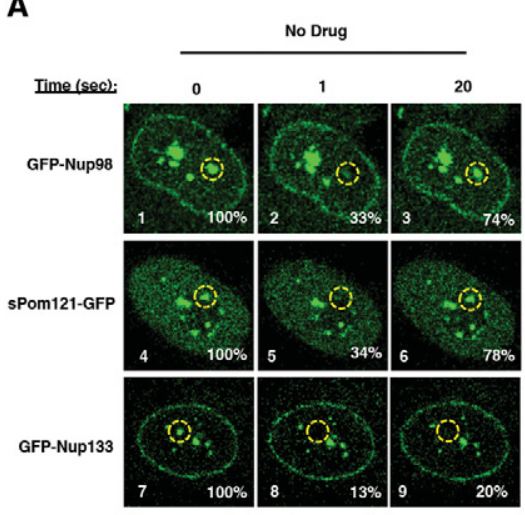

B

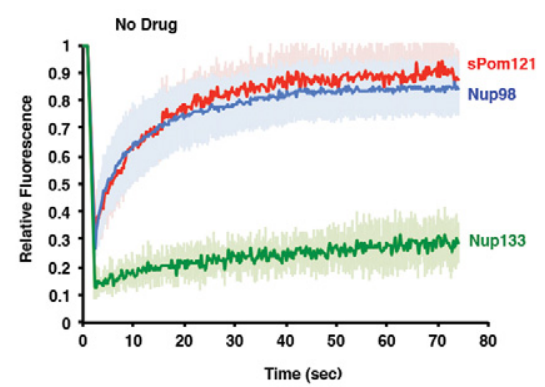

C

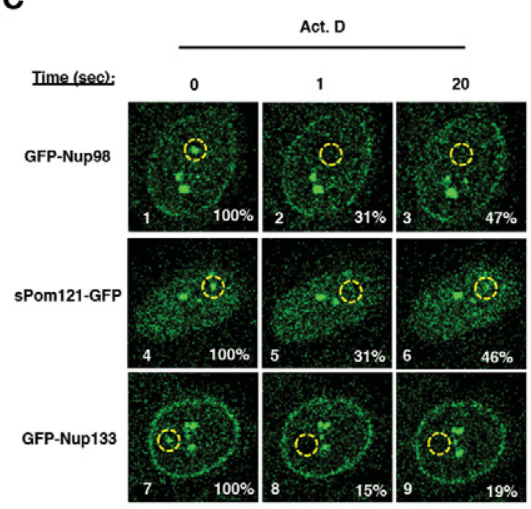

D

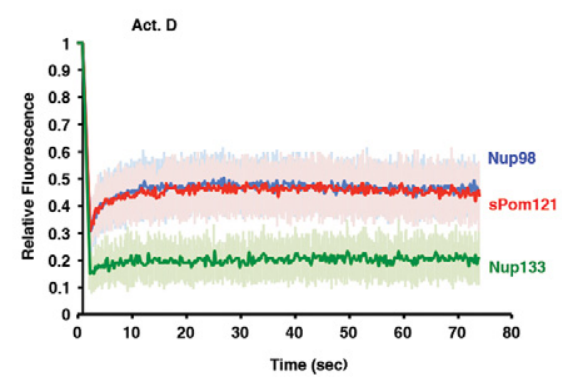

Figure 4. sPom121 and Nup98 have similar intranuclear dynamics. (A) FRAP assays showing fluorescence recovery of GFPNup98 (panels 1-3), sPom121-GFP (panels 4-6), or GFP-Nup133 (panels 7-9) during the time course shown at the top. The percent fluorescence recovery is indicated in the bottom right corner of each image, while the bleached area is indicated by the yellow dotted line. (B) Graph showing fluorescence recovery of either GFP-Nup98 (blue), sPom121-GFP (red), or GFP-Nup133 (green) under wild-type cell conditions. $(C)$ FRAP assays showing fluorescence recovery of GFP-Nup98 (panels 1-3), sPom121-GFP (panels 4-6), or GFP-Nup133 (panels 7-9) in the presence of the transcriptional inhibitor actinomycin $\mathrm{D}$ (Act. D) during the time course shown at the top. The percent fluorescence recovery is indicated in the bottom right corner of each image, while the bleached area is indicated by the yellow dotted line. $(D)$ Graph showing fluorescence recovery of either GFP-Nup98 (blue), sPom121-GFP (red), or GFP-Nup133 (green) in cells treated with actinomycin D.
sPom121 are different. To test this, we expressed GFPtagged Nup133, a component of the Nup107/160 complex that has also been shown to colocalize with Nup98 in nucleoplasmic foci (Morchoisne-Bolhy et al. 2015). Unlike Nup98 and sPom121, intranuclear GFP-Nup133 fluorescence recovers slowly following a photobleach (Fig. 4A [panels 8,9], B [green trace]) suggesting that sPom121 and Nup98 might be in a subcomplex distinct from Nup133 in the nucleoplasm. These results indicate that sPom 121 and Nup98 might have a shared function in some intranuclear process.

Previous studies demonstrated that Nup98 is a potent transcription factor in the nucleoplasm whose dynamics on chromatin are blocked by transcriptional inhibitors (Griffis et al. 2002, 2004; Capelson et al. 2010; Kalverda and Fornerod 2010; Pascual-Garcia et al. 2014). For example, when cells were treated with actinomycin D, the fluorescence recovery of Nup 98 by FRAP was much slower as Nup98 becomes immobilized on chromatin and unable to recycle to intranuclear foci (Griffis et al. 2002, 2004). We hypothesized that sPom121 cooperates with Nup98 to regulate transcription at Nup98 target genes and predicted that its dynamics would be similar to those of Nup98. To test this, we expressed either GFP-Nup98 or sPom121-GFP in HeLa-C cells and conducted FRAP assays in the absence or presence of actinomycin D. Remarkably, the fluorescence recovery observed for sPom121 matches the kinetics displayed by Nup98 under all conditions (Fig. 4D). In contrast, GFP-Nup133 displays different kinetics in the presence of actinomycin D (Fig. 4C,D). This suggests that sPom121 and Nup98 are likely part of the same complex, the dynamics of which are dependent on the transcription status of the cell.

\section{Nup98 and sPom121 cobind many promoters in human cells}

Nup98 was previously shown to bind to intranuclear gene promoters to regulate transcription (Capelson et al. 2010; Kalverda and Fornerod 2010; Liang et al. 2013; Light et al. 2013). This raised the interesting possibility that sPom121, via its loss of membrane anchoring and NPC targeting, might have acquired the ability to bind to Nup98 target genes independently of localization at the nuclear periphery. This would allow it to expand its transcriptional role beyond that of the original Pom121 protein. To address this, we attempted to determine the genome-wide binding pattern of $s$ Pom121 using a DamID (DNA adenine methyltransferase [DAM] identification) approach (van Steensel and Henikoff 2000). We fused a DAM tag to Nup98, sPom121, or a control protein GFP (Fig. 5A) and expressed them in human HeLa-C cells. After extraction, methylated DNA was processed and subjected to deep sequencing and mapping against the reference human genome assembly. Peaks that were significantly above GFP-DamID background levels were counted as positive hits (Fig. 5B).

Intriguingly, we observed significant cobinding of Nup98 and sPom121 at 1056 genomic sites, 492 of which were co-occupied promoters (Fig. 5C). As compared with other genomic elements, Nup98 and sPom121 DamID results revealed a strong preference for binding in close proximity to the transcriptional start sites of genes 
Franks et al.
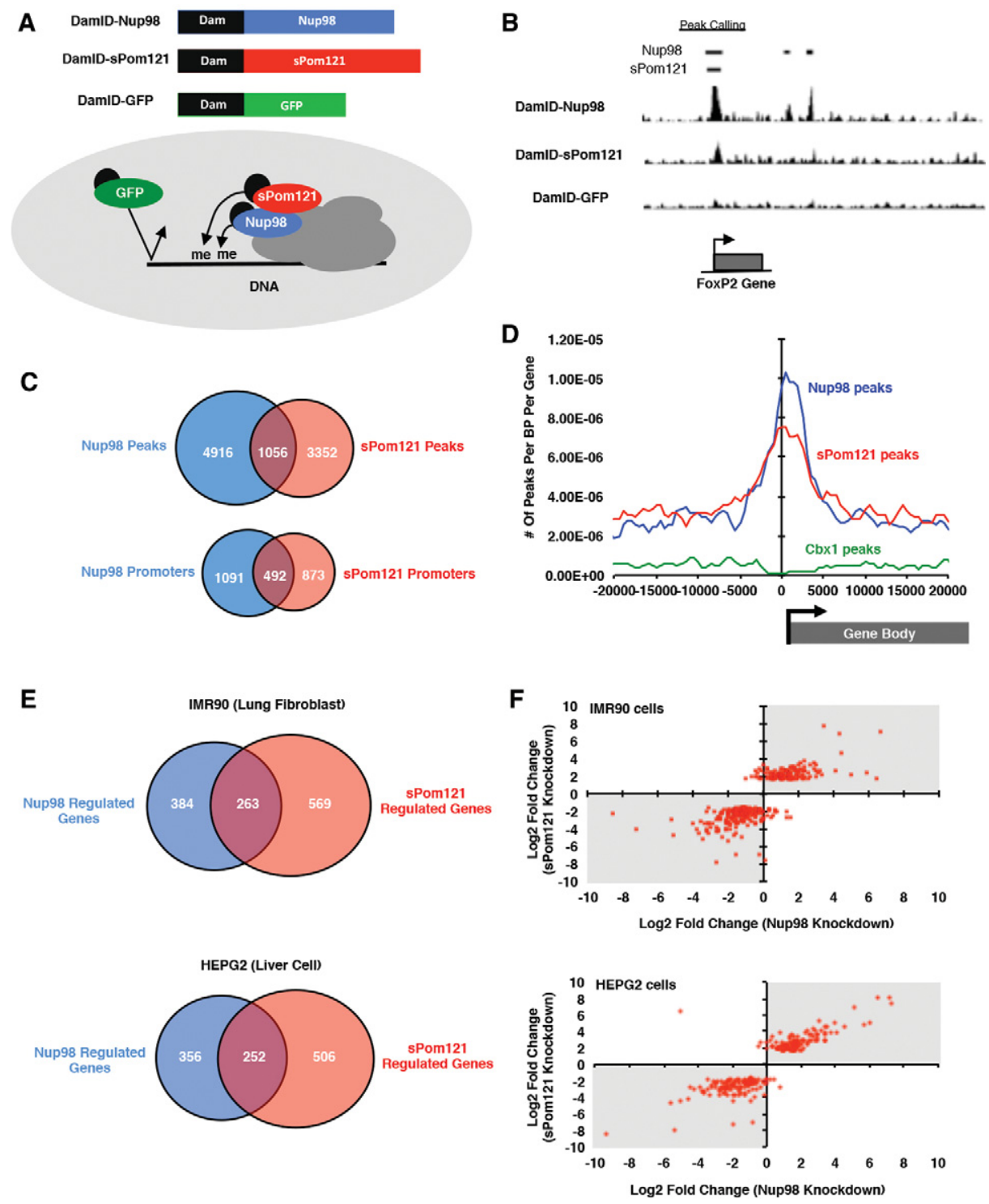

Figure 5. Nup98 and sPom121 cobind promoters in human cells. (A) Schematic of DamID constructs used to identify genomic binding sites of Nup98 (blue), sPom121 (red), and GFP (green). (Bottom diagram) DamID-tagged proteins that interact specifically with chromatin are expected to leave a well-defined peak, while GFP should not interact with chromatin and can be used to measure background DNA methylation. (B) Example of Nup98- and sPom121-binding peaks on a representative gene, FoxP2. Peaks that were called are shown at the top $(C)$ Venn diagram showing overlap of Nup98 (blue) and sPom121 (red) DamID peaks (top) and Nup98 and sPom 121 peaks that overlap at promoters (bottom) in HeLa-C cells. (D) Graph showing the number of peaks per base pair per gene relative to their transcriptional start sites. The $X$-axis represents location relative to the transcription start site (0 on the graph), while the $Y$-axis is the number of peaks per base pair per gene. Peaks identified in DamID experiments with Nup98 (blue), sPom121 (red), and a negative control protein, Cbx1 (green), which binds repressive chromatin are shown. (E) Overlap of genes misregulated by sPom121 (red) and Nup98 (blue) knockdowns. We asked what percentage of genes that are misregulated in sPom 121 data sets $(P$-value $<0.01)$ is also misregulated by Nup98 knockdown $(P$-value $<0.01)$. The red area of the graph represents genes that were misregulated by sPom 121 knockdown, while the blue represents genes that were misregulated by Nup98 knockdown. $(F)$ Graph showing the $\log _{2}$ fold change of genes significantly misregulated (adjusted $P$-value $<0.05$ ) in sPom 121 knockdown data sets (Y-axis) plotted versus the $\log _{2}$ fold change of those same genes in Nup98 knockdown data sets ( $X$-axis). If genes were up-regulated or down-regulated by both knockdowns, a red dot appears in the top right or bottom left quadrant of the graph, respectively (gray area of graph). In contrast, if a gene was misregulated by knockdown of either sPom121 or Nup98 but not by the other protein, a red dot appears in the top left or bottom right quadrant of the graph (white space). 
(Fig. 5D), which is similar to what has been shown previously for Nup 98 and is indicative of a role in transcriptional initiation (Capelson et al. 2010; Kalverda and Fornerod 2010; Liang et al. 2013). When we performed DamID experiments with a DamID-Nup98 mutant (DamIDNup98 CTD) that has dramatically reduced affinity for the NPC /Griffis et al. 2002; Kalverda and Fornerod 2010), we found that its chromatin-binding profile is very similar to sPom121 and wild-type Nup98 (Supplemental Fig. S3C). Of the 1056 regions bound by both Nup98 and sPom121, 580 of those peaks $(55 \%)$ were also bound by intranuclear-specific Nup98 CTD, suggesting that the majority of sPom121 targets are in the nucleoplasm.

To determine whether sPom121 and Nup98 affect the expression of their target genes, we depleted either sPom121 or Nup98 with siRNAs and observed the transcriptional consequences of this knockdown. For the former, we needed to specifically deplete sPom121 protein without altering levels of Pom121, which would reduce NPC number and potentially perturb nuclear transport. To achieve this, we targeted the sPom121 siRNA to exon 3 of the sPom121 5' UTR that is unique from the Pom121 mRNA (Supplemental Fig. S3D) and is not well conserved with the $5^{\prime}$ UTR of the RB-associated $K R A B$ zinc finger (RBAK) gene from which the sPom 121 5' UTR originated. As shown in Supplemental Figure S3E, qPCR results in HeLa-C cells showed that, while sPom121 RNA was efficiently depleted, as determined by the reduction of expression of the first 3 exons of sPom121 (left graph, red bar), levels of the Pom121 RNA remained stable, as measured by the levels of the TM-encoding exon 4 of Pom121 (right graph, red bar).

Having an experimental system in place to study the role of sPom121 in transcription without affecting nuclear transport or NPC density (Supplemental Fig. S4A,B), we focused on genes that were strongly bound by sPom 121 and Nup98. These included genes encoding transcription factors such as Myc and TFAP2A as well other targets involved in diverse cellular processes, such as cell signaling and Golgi apparatus function (Dkk1 and Ext1) (Supplemental Fig. S3A). qPCR results showed that many of the genes tested, including Myc, TFAP2A, Ext1, and $D k k 1$, were up-regulated by both sPom121 knockdown and Nup98 knockdown (Supplemental Fig. S3B), suggesting an important role for sPom121 in gene repression in HeLa-C cells. We also found a small number of target genes that were down-regulated in the presence of either the sPom121 or Nup98 siRNA, suggesting that sPom121-Nup98-dependent gene regulation can vary depending on the target. We therefore conclude that sPom121 and Nup98 cooperate to regulate the expression of target genes involved in diverse cellular processes in HeLa-C cells.

We next wondered whether sPom121 plays a role as a transcriptional regulator in cells derived from different tissues. To test this, we used siRNAs to deplete sPom121, Nup98, or a control protein (Fluc) in two different cell types: human IMR90 lung fibroblasts and HEPG2 human liver cells. We conducted gene expression analysis using RNA-seq technology. We found that many genes were significantly misregulated in IMR90 (832 genes) and HEPG2 (758 genes) cells in the presence of sPom121 siRNA $(P$-value $<0.01)$ (Fig. $5 \mathrm{E})$. We observed similar results in cells treated with Nup98 siRNA, where gene expression in IMR90 (647 genes misregulated) and HEPG2 (608 genes misregulated) cells was dramatically disrupted (Fig. 5E). Intriguingly, there is significant overlap between these two data sets; many of those genes that were misregulated in sPom121 knockdown cells were also misregulated by Nup98 knockdown in IMR90 (263 genes) and HEPG2 (252 genes) cell types (Fig. 5E, overlapping regions; Supplemental Figs. S5, S6). Importantly, genes were misregulated in the same direction when cells were treated with sPom121 siRNA or Nup98 siRNA (Fig. 5F, dots falling within the gray boxes indicate genes that changed in the same direction for both knockdowns). We conclude that sPom121 cooperates with Nup98 to regulate the expression of intranuclear genes in diverse cell types. Intriguingly, genes that are most misregulated in sPom121 and Nup98 knockdown cells are particularly important for homeostasis of the tissue in question (Supplemental Fig. S4C).

\section{Convergent evolution of sPom121-like homologs in mammals}

We demonstrated that sPom121 is produced in human cells via alternate transcriptional initiation that produces an mRNA with a $5^{\prime}$ UTR unique to the Pom121 mRNA. We wondered whether the alternative transcription start site and unique 5' UTR of sPom121 are highly conserved or whether these features are recently evolved. We analyzed the conservation of the unique sPom121 5' UTR in other metazoan species. Surprisingly, we found that the novel Pom121 5' UTR arose when four exons from the $R B A K$ gene, which encodes a retinoblastoma 1-binding protein, duplicated in a common ancestor of hominoids within the last 20 million-25 million years (Fig. $6 \mathrm{~A}$, bottom; gray exons $1-3)$. The $R B A K$ gene duplication is positioned close to the $5^{\prime}$ end of the Pom121 gene such that the $R B A K$ transcriptional start site became an alternate start site for Pom121 expression. The original $R B A K$ ORF is not conserved at the Pom121 locus, so these exons are now part of the 5' UTR of $s$ Pom 121. Additionally, as others have discussed previously (Kipersztok et al. 1995; Antonell et al. 2005; Funakoshi et al. 2007), we observed that the POM121 gene locus was duplicated after the acquisition of the $5^{\prime}$ UTR of $R B A K$, resulting in expression of Pom121 mRNAs from at least two different loci (Pom121 and Pom121C). The Pom121 and Pom121C loci appear to be functioning similarly to produce both Pom121 and sPom121 proteins, whereas an additional Pom121 duplication, Pom121B, does not appear to be active (Funakoshi et al. 2007). Although we focused the majority of our study on the Pom121 locus, we designed our experiments to ensure that any observation or manipulation of sPom121 or Pom121 expression affected both the Pom121 and Pom121C loci.

The genomic rearrangement that brought the $R B A K$ exons upstream of POM121 appears to be exclusive to the 
Franks et al.

A
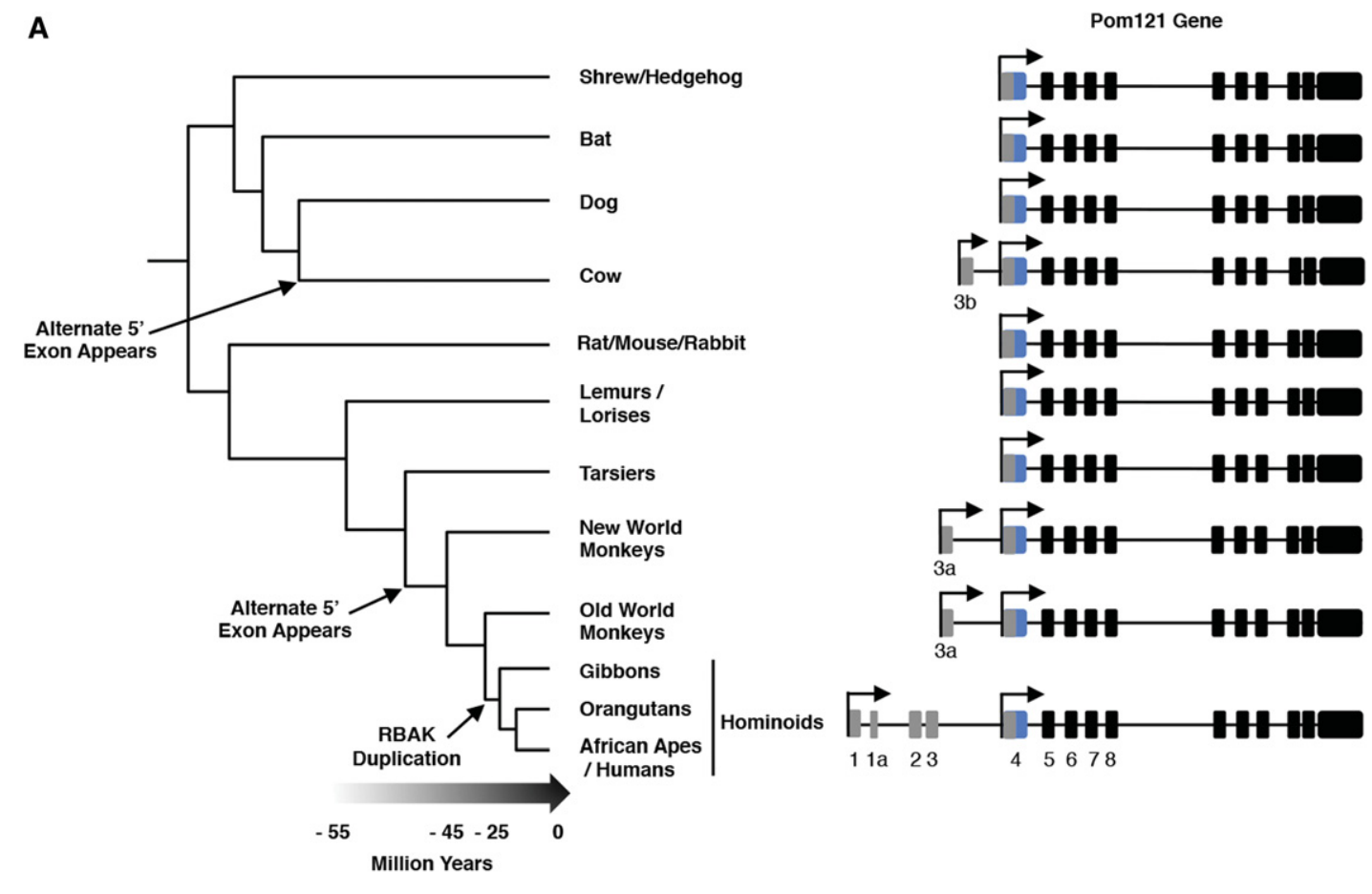

B

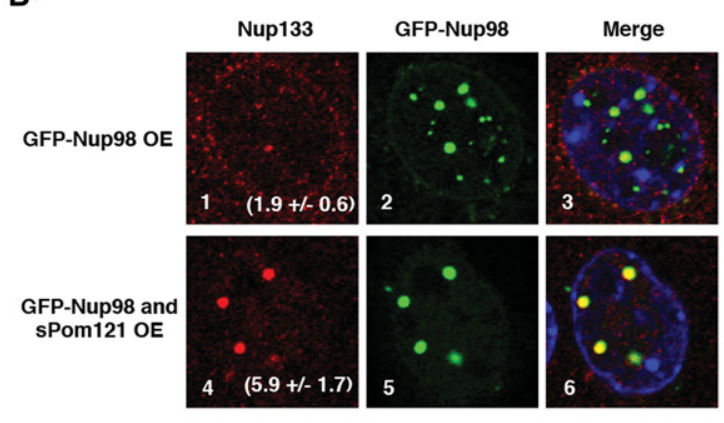

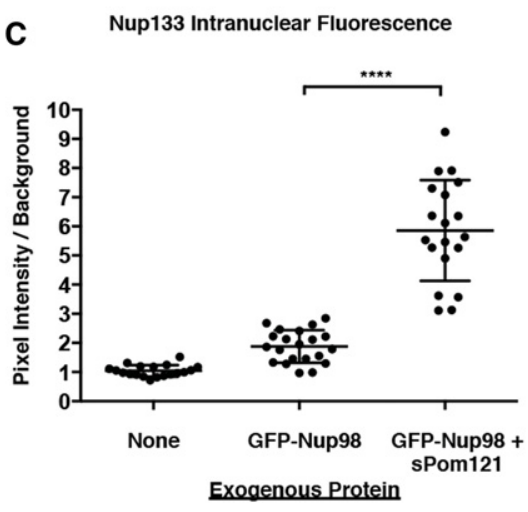

Figure 6. The hominoid version of sPom 121 evolved recently and can recruit the Nup107/160 complex to the nucleoplasm when expressed in nonhominoid cells. (A) Novel upstream exons acquired sporadically during mammalian evolution are indicated in gray, while the Pom121 TM domain-coding exon (exon 4) is shown in blue, and the exons coding for the Pom121 ORF are shown in black. Note that, for simplicity, we are not showing the hominoid-specific duplication of the Pom 121 gene (Pom121C). The gene structure of Pom $121 \mathrm{C}$ is similar to the Pom 121 locus, which is shown. (B) Localization of the endogenous Nup133 protein in mouse C2C12 cells in the presence of exogenous GFP-Nup98 or GFP-Nup98 and sPom121 together. The fold increase in Nup133 intensity in transfected cells relative to untransfected cells is shown in the bottom right corner of panels 1 and 4 . $(C)$ Plot of the fluorescence intensity of intranuclear Nup133. The intensity of Nup133 intranuclear foci in transfected cells was measured and plotted relative to Nup133 intranuclear staining in untransfected cells (background).

ancestral hominoid lineage. However, we found that an alternative Pom121 transcript lacking its TM domain has evolved on at least two other independent occasions during mammalian evolution. First, we identified ESTs from marmosets and rhesus macaques (New World and Old World monkey species, respectively) that correspond to alternative Pom121 transcripts that use an alternative 5'UTR that lacks the TM-coding exon of Pom121, just like hominoid sPom121 (Fig. 6A). However, in this case, only a single upstream exon is expressed (exon 3a), which again splices directly to the second canonical coding exon of Pom121 (exon 5), bypassing the TM-coding exon (exon 4). Notably, the alternative Pom121 exon in monkeys does not align with any sequence in hominoids. Together, these data suggest that the simian primate ancestor Pom121 gene first acquired a novel alternative transcription start site and an upstream exon encoding a sPom121 protein without a TM domain. Later, in the hominoid ancestor, the region containing that upstream exon was replaced by the $R B A K$-like exons, creating a 
different transcriptional isoform that nevertheless encodes the equivalent sPom121 protein. In addition to primates, we found EST evidence for a third, independent evolutionary recruitment of alternative upstream exons encoding the sPom121 protein in an ancestor of cows (Fig. 6A; "exon 3b"). Like in monkeys, the cow version of the alternative Pom121 transcript expresses only one novel upstream exon, but the sequence is not homologous to any sequence found in monkeys or hominoids. Nevertheless, it results in a transcript predicted to encode a sPom121-like protein.

We next wanted to test whether the sPom121 transcripts, which evolved independently in monkeys and cows, are also expressed like in humans. Using publicly available RNA-seq data from several different marmoset (New World monkey), rhesus macaque (Old World monkey), or cow tissue samples, we were able to observe expression of the noncanonical sPom121 exon but only in the testes of marmosets and rhesus monkeys (Supplemental Fig. S7A,B). No sPom121 expression was observed in the cerebellum, heart, kidney, or liver (Supplemental Fig. S7A,B). In cows, we found no evidence for expression of $s$ Pom121 (brain, kidney, and liver tissues examined) (Supplemental Fig. S7C). These expression results strongly contrast with those in humans, in which we observed abundant sPom121 mRNA in all tissues tested (Fig. 1D). This might reflect the better sampling of tissue types in humans relative to other primates or cows. Nevertheless, our findings highlight that alternate sPom121-like proteins have emerged via convergent evolution at least three times during mammalian evolution.

Interestingly, the widespread sPom121 expression in hominoids seems to correlate with the appearance of nucleoplasmic Nup107/160 complexes that are not associated with the NE (Morchoisne-Bolhy et al. 2015). Since a major role of Pom121 is to recruit the Nup107/160 complex to the NE during NPC assembly (Antonin et al. 2005; Doucet et al. 2010; Mitchell et al. 2010; Funakoshi et al. 2011; Talamas and Hetzer 2011), we predicted that sPom121 (which lost its ability to integrate into the NE) might be able to redirect/retarget NPC scaffold proteins to non-NE locations in the nucleus. To test this idea, we expressed GFP-Nup98 to induce the formation of Nup98 foci that are not associated with the NE in mouse C2C12 cells that do not express sPom121. As expected for cells that do not express sPom121, we observed Nup133 (a component of the Nup107/160 complex) at NPCs but not strongly enriched at Nup98 foci (Fig. 6B [panel 1], C). In contrast, ectopic expression of human sPom121 resulted in the significant recruitment of Nup133 to Nup98 foci (Fig. 6B [panel 4], C). No Nup133 intranuclear foci were observed in cells expressing sPom121 alone (data not shown) possibly because intranuclear Nup98 is limiting under these conditions. Our data suggest that sPom121 along with Nup98 is sufficient to target the Nup107/160 complex to non-NE sites. We conclude that in cells lacking SPom121 (prior to hominoids), Nup98 is not sufficient to recruit a significant amount of Nup107/160 to the nucleoplasm (Fig. 7A [left side], B [left side]). However, in hominoid cells, sPom121 is expressed and can compete with Pom121 for recruitment of Nup107/160 complexes during the initial stages of mitotic and interphase NPC assembly (Fig. 7A [right side], B [right side]). Moreover, our results suggest that the recent appearance of SPom121 might represent a crucial step in the functional evolution of not just Pom121 but also the Nup107/160 complex.

\section{Discussion}

sPom121 is a novel transcription cofactor for Nup98mediated gene regulation

In this study, we provide evidence that a soluble form of Pom121 exists in human cells that, together with Nup98, has the ability to bind to specific promoters to
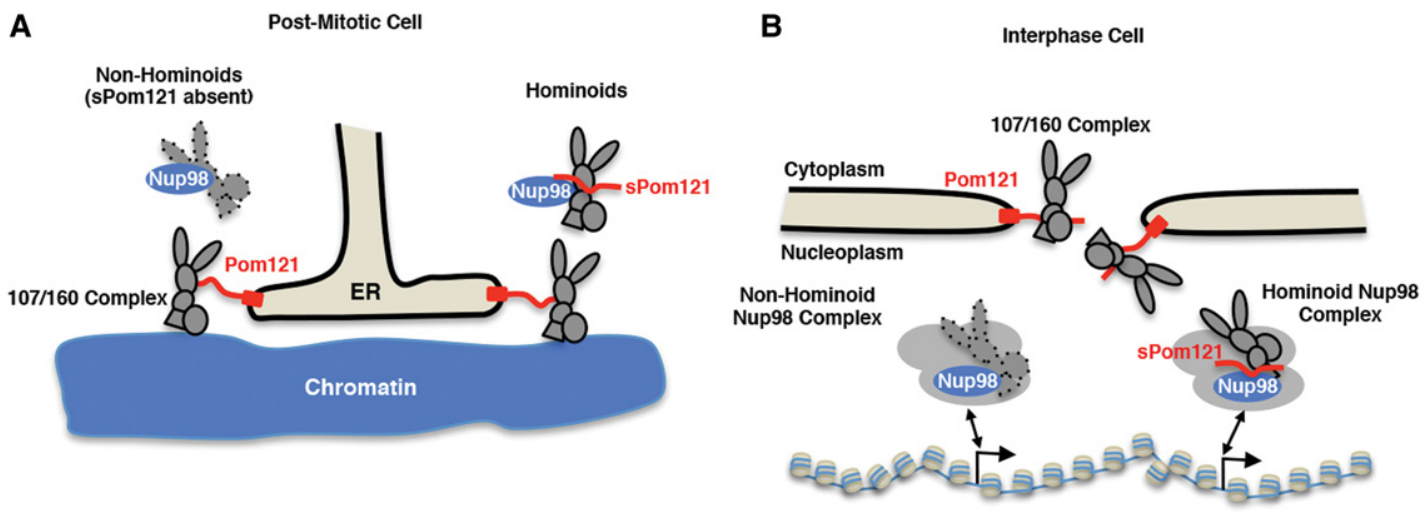

Figure 7. Model for sPom121-mediated recruitment of the Nup107/160 complex to the nucleoplasm of hominoid cells. (A,B, left side of model) In nonhominoid cells, sPom 121 is likely not present, resulting in very little Nup133 (Nup107/160 complex) being prevented from entering the NPC during post-mitotic NPC assembly. (A,B, right side of model) In hominoids, sPom121 interacts with Nup98 and increases the affinity of Nup133 (Nup107/160) for Nup98 intranuclear complexes. As a result, a significant amount of Nup133 is retained in the nucleoplasm during post-mitotic and interphase NPC assembly. It remains to be seen whether the Nup107/160 complex can bind to Nup98 target genes and whether this has an effect on the regulation of said genes. 
regulate their transcriptional output. Without its TM domain, the localization of sPom121 and its functional properties become distinct from those of Pom121. Its nucleoplasmic localization allows sPom121 to explore the entire genome regardless of nuclear membrane proximity. We show that sPom121 colocalizes with Nup98 and the Nup107/160 complex via its NLS domain in an NPCindependent manner (Figs. 2B, 3B; Mitchell et al. 2010), thereby uncoupling transcription control from NPC function. sPom121 and Nup98 have nearly identical transcription-dependent dynamics in the nucleoplasm, suggesting that they mobilize in the nucleus in the same protein complex, which likely plays a direct role in transcription (Fig. 4A,B). Indeed, we found that sPom121 and Nup98 cobind to many gene promoters (Fig. 5C,D; Supplemental Fig. S3) in the nucleoplasm; knockdown of either protein misregulates an overlapping set of genes (Fig. 5E,F; Supplemental Figs. S5, S6). Although gene ontology results revealed some similarity among gene clusters that are misregulated across all cell types in the absence of sPom121 and Nup98, our data suggest that sPom121 regulates different gene sets depending on the cell type (Supplemental Figs. S4C, S5, S6). Overall, those genes most highly misregulated by sPom 121 depletion are usually also misregulated in the same direction by Nup98 knockdown (Fig. 5E,F), offering further evidence that sPom121 is cooperating with Nup98 to regulate transcription.

\section{What is the role of sPom121 in mammalian cells?}

What unique advantage is provided by the presence of sPom121 that improves the ability of Nup98 to regulate transcription in hominoids, monkeys, and cows? Recent studies in yeast have demonstrated the ability of the NPC to promote gene looping at the NPC, which allows rapid reinitiation of transcription after brief periods of repression (Casolari et al. 2004; O'Sullivan et al. 2004; TanWong et al. 2009; Brickner and Brickner 2011). Furthermore, other studies have demonstrated that the NPC is an important scaffold at the nuclear periphery, where active genes and transcription factors congregate (Raices and D'Angelo 2012; Ptak et al. 2014; Ibarra and Hetzer 2015). The ability of the NPC to regulate gene expression is confined by its peripheral localization in yeast. Thus, it seems that evolution would favor a more mobile subset of Nups that can regulate gene expression anywhere in the nucleus, even beyond the NPC. Indeed, a subset of peripheral Nups like Nup98 has acquired the ability to move off the NPC in metazoan species such as Drosophila (Capelson et al. 2010; Kalverda and Fornerod 2010). Interestingly, however, other than Sec13, no scaffold Nups have been shown to localize in the nucleoplasm in Drosophila cells, implying that gene expression regulation probably does not occur via an NPC-like scaffold in nonmammalian species but instead involves recruitment of chromatin-modifying complexes to chromatin by individual Nups (Capelson et al. 2010; Pascual-Garcia et al. 2014). The recent finding that the Nup107/160 scaffold can exist away from the NPC in human cells raises the possibility that larger Nup subcomplexes probably exist in the nucleoplasm in mammalian cells (Morchoisne-Bolhy et al. 2015).

Our findings that even the TM Nup Pom121 has evolved to be expressed in a soluble form provides further supporting evidence for the evolution of an intranuclear NPC subcomplex. Interestingly, Pom121 protein was previously shown to interact with both Nup98 and the Nup107/160 complex, raising the possibility that sPom121 promotes the interaction between Nup98 and the Nup107/160 complex in the nucleoplasm (Mitchell et al. 2010). Previous studies have demonstrated that Pom121 promotes recruitment of the Nup107/160 complex to chromatin as well as the interaction between nascent pores and reforming nuclear membranes during the early stages of post-mitotic NPC assembly (Fig. 7A, B). It is possible that sPom121 competes with membrane-bound Pom121 for interaction with the Nup107/ 160 complex to promote prevention of a fraction of the Nup107/160 complex from entering NPCs (Fig. 7A,B). Indeed, we demonstrated that, in contrast to human cells, Nup107/160 complexes are not efficiently recruited to the nucleoplasm of mouse $\mathrm{C} 2 \mathrm{C} 12$ cells. However, if exogenous sPom121 is expressed, the Nup107/160 complex (Nup133) is strongly recruited to Nup98 complexes (Fig. $6 \mathrm{~B}, \mathrm{C})$. These findings support the idea that nucleoplasmic localization of the Nup107/160 complex simultaneously appeared with the evolution of sPom121. It will be interesting to investigate whether the Nup107/160 complex can bind and play a role in the regulation of Nup98/ sPom121-bound genes, which would provide further evidence for the assembly of an off-pore NPC scaffold functioning in gene regulation at intranuclear promoters.

\section{The Pom121 locus is rapidly evolving}

One remarkable aspect of the Pom121 gene is the hominoid-specific gene duplication and fusion with regulatory elements of the $R B A K$ gene that resulted in the use of an alternative transcriptional start site (Fig. 6A). After Pom121's appearance in vertebrates, it quickly gained an essential role in the regulation of interphase NPC assembly (Doucet et al. 2010; Dultz and Ellenberg 2010; Funakoshi et al. 2011; Talamas and Hetzer 2011; Field et al. 2014). The Pom121 locus remained almost unchanged after this vertebrate-specific innovation (Antonell et al. 2005) until the divergence of mammals, where the expression of sPom121 appears to have evolved at least three different times. What does this tell us about the importance of the sPom121 protein? If convergent evolution is at play, it suggests that an important fitness benefit is provided by sPom121 in not just human cells as we demonstrated but possibly other mammalian species. Unlike human cells, we did not see any expression of sPom121 mRNA in monkeys outside of the testis or clear evidence that sPom 121 is expressed at significant levels in cows. Taken at face value, these expression studies suggest that the drastic genomic rearrangement of the Pom 121 gene in hominoids may have allowed for more widespread expression of sPom 121 than in other species. In the cow and monkey versions of sPom121, only one upstream exon is present, which arose 
from an unknown origin. In contrast, in hominoids, duplication of the $R B A K$ gene resulted in the addition of three or four exons to the $5^{\prime}$ end of the Pom121 gene and importantly donated a functional promoter (Fig. 1C), which might have been a breakthrough toward more ubiquitous sPom121 expression.

Prior to hominoid divergence, the entire Pom121 gene duplicated to form the Pom121C gene (Funakoshi et al. 2011). It is currently unclear whether the Pom121 and Pom121C genes produce proteins with redundant functions. Interestingly, there are many amino acid changes as well as several insertions/deletions that have arisen since the duplication of Pom121C, which will give rise to a protein that differs considerably in amino acid sequence from that of the sPom121 protein derived from the Pom121 gene. In the future, it will be interesting to closely analyze the function of $s$ Pom121 and Pom121 proteins derived from the two Pom121 loci to see whether they differentiated further in function. In turn, this might provide further insights into Nup-mediated transcriptional regulation and how it evolves.

In summary, we provide evolutionary and cell-biological evidence for the presence of a previously undetected soluble form of the TM Nup Pom121, which plays a dedicated role in transcription control. This transcription regulator contains all features of the NPC-associated Pom121 except that it lacks the nuclear membrane-anchoring and NPC targeting domain. sPom121 arose in the last common ancestor of hominoids from a rearrangement of genomic sequences that generated a new transcriptional start site, exhibiting a classic example of what Jacob (1977) called "molecular tinkering." According to his idea, the appearance of a new molecular function is generated by alterations of pre-existing ones. In the context of the $\mathrm{NPC}$, it appears that the adaptive advantage of generating sPom121 as a soluble NLS-containing regulator appears to lie in the potential uncoupling of Nup-mediated gene regulation from the spatial constraints of NPC-mediated genome regulation at the nuclear periphery. This uncoupling allows the expansion of Pom121's role in transcriptional regulation without compromising its role at the NPC, providing an elegant demonstration of the relief from the inherent antagonistic pleiotropy of encoding two important functions in the same Nup protein.

\section{Materials and methods}

\section{IF and antibodies}

HeLa-C cells (a gift from Volker Cordes and Maureen Powers) were cultured in DMEM/10\% fetal bovine serum (FBS)/penicillinstreptomycin at $37^{\circ} \mathrm{C}$ in $5 \% \mathrm{CO}_{2}$. For IF assays, cells were washed once with $1 \times$ phosphate-buffered saline (PBS) and fixed for $2 \mathrm{~min}$ in $4 \%$ paraformaldehyde (PFA). After three washes with PBS, cells were incubated for $10 \mathrm{~min}$ in $1 \times$ IF buffer $(10 \mathrm{mg} / \mathrm{mL}$ BSA, $0.1 \%$ Triton X-100, $0.02 \%$ sodium dodecyl sulfate [SDS], $1 \times$ PBS). Next, cells were incubated for $2 \mathrm{~h}$ in IF buffer containing the antibodies of choice. The antibodies used included mouse Nup98 (1:100 dilution; Santa Cruz Biotechnology, C-5) (Fig. 2; Supplemental Fig. S2), rabbit Nup98 (1:500 dilution; Cell Signaling, P671) (Supplemental Fig. S2C, panels 2, 17,23), Pom121 amino acids 236-552 (1:500 dilution [for IF] or 1:1000 dilution [for Western blot]; Genetex, GTX102128), Pom121 amino acids 1214-1229 (1:250 dilution; produced in the Hetzer laboratory), Pom121 amino acids 448-647 (1:500 dilution; produced in the Hetzer laboratory), rabbit Nup96 (1:500 dilution; NB100-93325), rabbit Nup133 against amino acids 1-22 (1:500 dilution; produced in the Hetzer laboratory), rabbit Nup107 (1:500 dilution; produced in the Hetzer laboratory), Elys (1:1000 dilution; produced in the Mattaj laboratory), Nup153 (1:1000 dilution; gift from B. Burke), TPR (1:500 dilution; Abcam, G00198), Nup50 (1:500 dilution; Abcam, G00318), Nup214 (1:1000 dilution; produced in the Hetzer laboratory), Nup358 (1:1000 dilution; Bethyl Laboratories, A301-796A), Nup88 (1:1000 dilution; BD Biosciences, 611896), Nup93 amino acids 2-218 (1:500 dilution; produced in the Hetzer laboratory), mAB414 (1:1000 dilution; Biolegend MMS-120R), and tubulin (1:1000 dilution [Western blot]; Sigma, T5168).

\section{Plasmids}

All sPom 121 constructs were cloned from a rat Pom 121 construct that has been described previously (Doucet et al. 2010; Talamas and Hetzer 2011). Importantly, this rat Pom121 construct has been shown to function similarly to human Pom 121 in all assays tested (Doucet et al. 2010; Talamas and Hetzer 2011). rPom121$3 x$ GFP was made by amplifying rPom121 amino acids 1-1200 and ligating the N-terminal of a 3xGFP tag. sPom121-GFP and fragments thereof were made by amplifying the DNA fragments corresponding to the amino acids indicated in the figures (amino acid 1 of sPom 121 corresponds to amino acid 241 of rat full-length Pom121) by PCR primers containing gateway-compatible sites. sPom121-Flag (Fig. 4B) was constructed by using a reverse oligo with a C-terminal Flag tag to amplify sPom121. Oligos also contained Gateway recombination sites that allowed for recombination into the pDonr207 vector. A subsequent recombination step was used to recombine sPom121-Flag into the pQXCIB retroviral vector. This vector was cotransfected with GFP-Nup98 (Fig. 4B). GFP-Nup98 was a gift from Jan Ellenberg. GFPmNup133 has been described previously (Talamas and Hetzer 2011). PCR fragments were recombined into Donr207 and subsequently recombined into pDest47. The vector used for DamID was made by PCR-amplifying and ligating the heat-shock promoter, N-terminal Eco-Dam tag, V5-tag, and gateway recombination sites (RFC.1) from the pLgw-EcoDam-V5-RFC vector (a gift from B. van Steensel's laboratory) in to the polylinker of the pMSCVpuro retroviral construct (pMSCVpuro-DAMID). cDNA corresponding to hNup98 amino acids $1-863$, hNup98 amino acids 1-504 (DamID-Nup98 $\Delta$ CTD), rPom121 amino acids 241-1200, or GFP were recombined into the pDonr207 vector and subsequently recombined into the RFC region of the pMSCVpuro-DAMID vector.

\section{Detergent extraction of sPom121}

HeLa-C cells were grown in $15-\mathrm{cm}$ plates with or without Pom121 siRNA. Twenty-five million cells (usually a confluent 15-cm dish of HeLa-C cells) per sample were used to extract sPom121 and subsequently Pom121. For sPom121 extraction, cells were washed with $1 \times$ PBS. Next, cells were placed in 10 $\mathrm{mL}$ of PBS and subsequently removed from the plates with a cell scraper. After spinning the cells in $10 \mathrm{~mL}$ of conicals for 5 min at $4^{\circ} \mathrm{C}$, PBS was removed, and cells were incubated in $1 \mathrm{~mL}$ of mild extraction buffer $(0.2 \%$ NP- $40,10 \mathrm{mM}$ Tris $7.5,150$ $\mathrm{mM} \mathrm{NaCl}, 1 \mathrm{mM}$ EDTA, protease inhibitors) for 25 million cells for $2 \mathrm{~min}$. Next, cells were spun at 3000 relative centrifugal force (rcf) for $3 \mathrm{~min}$ at $4^{\circ} \mathrm{C}$ to pellet insoluble material. Soluble nuclear lysates were removed and placed in a separate tube, of which 200 
$\mu \mathrm{L}$ of lysate was placed with $200 \mu \mathrm{L}$ of $2 \times$ SDS sample loading buffer and used as the soluble sample on Western blot. To extract membrane-bound components, including Pom121, insoluble pellets remaining after soluble protein extraction were incubated with $2 \mathrm{~mL}$ of harsh lysis buffer $(0.2 \%$ NP-40, $0.25 \%$ sodium deoxycholate, $0.05 \%$ SDS, $10 \mathrm{mM}$ Tris 7.5, $150 \mathrm{mM}$ $\mathrm{NaCl}, 1 \mathrm{~mm}$ EDTA, protease inhibitors) for 2 min. Samples were spun at 10,000 rcf for $5 \mathrm{~min}$, and $200 \mu \mathrm{L}$ of supernatant was combined with $200 \mu \mathrm{L}$ of $2 \times$ SDS load buffer. This sample was used as the membrane-bound fraction in Western blot.

\section{Detection of sPom121 by Western blot}

Lysates obtained from mild detergent extraction were run on $4 \%-$ $12 \%$ gradient gels at least until the point at which the protein marker corresponding to $\sim 75 \mathrm{kDa}$ ran off the bottom of the gel. This allowed maximum separation between bands running at higher molecular weights (i.e., separating full-length Pom 121 from sPom121). Following transfer of SDS gels to Western blots, cells were probed overnight at $4^{\circ} \mathrm{C}$ with Pom 121 antibody (1:1000 dilution; Genetex, GTX102128). Western blots were developed using supersignal pico chemiluminescent substrate (Thermo Scientific). Alternatively, for a cleaner Western blot of sPom121, a coimmunoprecipitation of sPom121 could be conducted. After mild detergent extraction of sPom121 from 25 million cells, $5 \mu \mathrm{L}$ of Pom 121 antibody was added to soluble lysate and incubated $4 \mathrm{~h}$ with rotation at $4^{\circ} \mathrm{C}$. Twenty-five microliters (bead volume) of protein A sepharose was added, and samples were incubated with rotation for $>2 \mathrm{~h}$ at $4^{\circ} \mathrm{C}$. Beads are then washed five times with mild lysis buffer and collected by centrifugation after each wash. After aspirating the last wash, $25 \mu \mathrm{L}$ of $2 \times$ SDS load buffer was added. Beads were boiled for $3 \mathrm{~min}$ and loaded onto SDS-PAGE.

\section{5' RACE to clone Pom121 mRNAs}

Two million cells of the specified cell type were pelleted by centrifugation and lysed with buffer RLT from the Qiagen RNAeasy kit. RNA was subsequently purified using the RNAeasy protocol. Five micrograms of RNA from each sample was used in a cDNA reaction to specifically amplify $5^{\prime}$ ends of Pom 121 mRNAs using SuperScript II reverse transcriptase (Life Technologies). The oligo used for Pom121 reverse transcription anneals to a common region shared between sPom121 and Pom 121. The oligo sequence was 5'-TTTCTCTTCCAGAGCTGTGAGATGCC-3'. Next, an anchor primer was ligated to the $3^{\prime}$ end of the Pom121 cDNA products $(4 \mu \mathrm{L}$ of Pom $121 \mathrm{cDNA}, 2 \mu \mathrm{L}$ of phosphorylated anchor primer, $2 \mu \mathrm{L}$ of RNA ligase buffer, $8 \mu \mathrm{L}$ of $50 \%$ PEG $8000,1 \mu \mathrm{L}$ of $10 \mathrm{mM}$ ATP, $1 \mu \mathrm{L}$ of $0.1 \mathrm{M} \mathrm{DTT}, 1 \mu \mathrm{L}$ of SS RNA ligase 1 [New England Biolabs]) overnight at $25^{\circ} \mathrm{C}$. The anchor primer sequence was $5^{\prime}$-TTTAGTGAGGGTTAATAAGCGGCCGCGTCGTGAC TGGGAGCGC-3' amine. Next, a primer that anneals to Pom 121 and a primer that anneals to the anchor primer were used to amplify Pom $1215^{\prime}$ cDNA fragments. Both primers contain 5' gateway recognition sites for subsequent cloning into pDonr207. The sequences of these primers were Pom 121 RACE reverse (5'-GGGGACCACTTTGTACAAGAAAGCTGGGTCCTCTGA GAATTGAGGCCTCTCTTCAG-3') and anchor PCR forward (5'-GGGGACAAGTTTGTACAAAAAAGCAGGCTTCGCGGCC GCTTATTAACCCTCACTAAA-3'). After bacterial transformation, colonies were picked into $\mathrm{LB}$ and grown overnight at $37^{\circ} \mathrm{C}$. DNA was purified using the Qiagen miniprep kit. Primers that anneal to the pDonr207 vector upstream of and downstream from the recombined product were used to sequence RACE clones. For evolutionary analysis (Fig. 4A), the 5' UTR of sPom121 identified by RACE was blasted against other mammalian species to determine the first appearance of the RBAK exons in the locus.

\section{Knockdown of sPom121 and detection of sPom121 by qPCR}

To obtain an efficient and specific knockdown of sPom 121 mRNA, we designed an siRNA that targets the $5^{\prime}$ UTR that is unique to sPom 121 and is not shared with Pom 121 mRNA. Importantly, the sequence of this siRNA targets all sPom 121 mRNAs regardless of whether they originate from the Pom 121 or Pom 121C locus. We also verified by RNA-seq that this siRNA does not target the mRNA of the Pom-ZP3 fusion. The target siRNA sequences used were sPom 121 siRNA sense $\left(5^{\prime}\right.$-GCAA CUUGCCCAAGUCCUUTT- $3^{\prime}$ ) and sPom121 siRNA antisense (5'-AAGGACUUGGGCAAGUUGCTT-3'). Importantly, this siRNA targets a region of the sPom121 UTR that shares poor sequence homology with RBAK. To detect sPom 121 by qPCR, we used a forward primer that anneals to the $5^{\prime}$ UTR sequence of sPom 121 and a reverse primer that anneals to exon 5 of the Pom 121 mRNA downstream from the TM-coding exon (see the Supplemental Material for sequences). For qPCR detection of sPom121 expression in 20 tissue types (Fig. 1D), we synthesized cDNA from RNA samples (Clontech, 636643) using SuperScript II reverse transcriptase (Life Technologies). Samples were subjected to qPCR using primers that specifically detect sPom 121 or the TM domain of Pom121. Expression was normalized to actin.

\section{FRAP experiments}

HeLa-C cells were transfected with sPom121-GFP (rPom121 amino acids 241-1200), GFP-Nup98 (a gift from Jan Ellenberg), or mNup133-GFP. After $48 \mathrm{~h}$, cells were placed in an imaging chamber to simulate natural growth conditions $\left(37^{\circ} \mathrm{C}\right.$ and $\left.5 \% \mathrm{CO}_{2}\right)$. A single focus was chosen and bleached to $<30 \%$ initial fluorescence. Recovery of fluorescence was followed for $70 \mathrm{sec}$. For each time point, the average fluorescence from at least 20 cells was used to calculate a final measurement.

\section{DamID experiments}

cDNA corresponding to sPom121, Nup98, Nup98 CTD, or GFP was cloned into MSCV-DamID-Gateway using the gateway system. Five micrograms of the MSCV-DamID vector of choice and $5 \mu \mathrm{g}$ of Ampho were cotransfected into 293T cells. After 2 $\mathrm{d}$, the medium was collected from the $293 \mathrm{~T}$ cells and placed onto HeLa-C cells for $6 \mathrm{~h}$ in the presence of polybrene. After 48 $\mathrm{h}$, cells were placed in medium containing $2 \mu \mathrm{g} / \mathrm{mL}$ puromycin. After 7-10 d, stable cell lines were obtained. DNA was harvested from 2 million cells using the Qiagen DNAeasy blood and tissue kit (catalog no. 69504). DNA $(2.5 \mu \mathrm{g})$ was digested in a $20-\mu \mathrm{L}$ reaction containing $1 \mu \mathrm{L}$ of Dpn 1 enzyme (New England Biolabs) and NEB buffer 4 at a $1 \times$ concentration for $>4 \mathrm{~h}$. The Dpn1-digested DNA was next ligated with the DamID adapter primer duplex. The sequences of the adapter duplex were AdR top (5'-CTAA TACGACTCACTATAGGGCAGCGTGGTCGCGGCCGAGG A-3') and AdR bottom (5'- TCCTCGGCCG-3'). To make the AdR duplex, 200 pmol of each primer were placed in an Eppendorf tube together, and the sample was diluted such that each primer was at a final concentration of $20 \mathrm{pmol} / \mu \mathrm{L}$. The duplex was next heated for $3 \mathrm{~min}$ at $95^{\circ} \mathrm{C}$ and allowed to slowly cool for $15 \mathrm{~min}$ to room temperature. The resulting DNA duplex was placed in a ligation reaction with the Dpn1-digested DNA (20 $\mu \mathrm{L}$ of Dpn1-digested sample, $3 \mu \mathrm{L}$ T4 ligase buffer [New England Biolabs], $3 \mu \mathrm{L}$ of AdR duplex primers, $4 \mu \mathrm{L}$ of $\mathrm{ddH}_{2} \mathrm{O}$ ) for $>4 \mathrm{~h}$ at $16^{\circ} \mathrm{C}$. Five microliters of the ligation sample was 
amplified by PCR with KOD DNA polymerase (Toyobo) using the manufacturer's guidelines. The primer used to amplify the DNA fragments was bio-Adr-PCR ( $5^{\prime}$ bio-GGTCGCGGCCGA GGATC- $3^{\prime}$ ). Note that the $5^{\prime}$ end of this primer contains a biotin label used in later purification steps. The resulting amplified DNA was diluted to $250 \mu \mathrm{L}$ in binding and washing buffer (5 $\mathrm{mM}$ Tris-HCL at pH 7.5, 0.5 mM EDTA) and sonicated in Eppendorf tubes for 15 min using a Bioruptor sonicator ( 15 cycles of 30 sec on/30 sec off). The size of sonicated DNA was verified to be 200-500 bp before moving to the next step. Next, biotinylated ends were pulled down using Invitrogen Dynabeads MyOne Streptavidin T1. Fifty microliters was washed three times in binding and washing buffer containing $1 \mathrm{M} \mathrm{NaCl}$. Next, the sonicated DNA was added to the $50 \mu \mathrm{L}$ of washed Dynabeads. $\mathrm{NaCl}$ was added to the sample at this step to bring the concentration to $1 \mathrm{M}$. The DNA-bead mixture was rotated for $30 \mathrm{~min}$ at $4^{\circ} \mathrm{C}$. The beads were then washed three times with binding and washing buffer $+1 \mathrm{M} \mathrm{NaCl}$. The bound DNA was digested from the beads in $20 \mu \mathrm{L}$ of DpnII digestion reaction for $2 \mathrm{~h}$ at $37^{\circ} \mathrm{C}$. The sample was cleaned using a Qiagen MinElute Reaction CleaNup kit. The DNA was eluted in $20 \mu \mathrm{L}$, and libraries were prepared according to Illumina's specifications.

\section{RNA-seq analysis}

The cell line indicated was transfected using RNAiMax (Invitrogen) and the desired siRNA at a final concentration of $25 \mathrm{nM}$. After $72 \mathrm{~h}$, cells were washed in $1 \times$ PBS, and RNA was isolated using the RNeasy (Qiagen) purification kit. Libraries were prepared using the Illumina RNA library preparation kit. Reads were aligned to the human genome (hg19, GRCh37) using STAR (version 2.2.0. c) (Dobin et al. 2013). Only reads that aligned uniquely to a single genomic location were used for downstream analysis (mapping quality score $[\mathrm{MAPQ}]>10$ ). Gene expression values were calculated for annotated RefSeq genes using HOMER by counting reads that overlapped exons (Heinz et al. 2010). Differentially expressed genes were found using EdgeR (Robinson et al. 2010). Genes that were significantly misregulated by sPom 121 depletion (Fig. 5E $[P$-value $<0.01], \mathrm{F}$ [adjusted $P$-value $<0.05]$ ) were used for analysis. They were compared with genes that were misregulated by Nup98 knockdown $(P$-value $<0.01)$. Gene ontology functional enrichment analysis was performed using DAVID (Dennis et al. 2003).

Marmoset (GSE50747; WUGSC 3.2/calJac3), rhesus macaque (GSE30352; BGI CR_1.0/rheMac3), and cow (GSE43013; Baylor Btau_4.6.1/bosTau7) RNA-seq reads were aligned to their respective genomes. These data were obtained from previous studies (Brawand et al. 2011; Cortez et al. 2014; Fushan et al. 2015).

\section{ChIP-seq}

Chromatin was fixed, and ChIP-seq was preformed as described previously (Liang et al. 2013; Jacinto et al. 2015). The antibody for H3K4me3 ChIP was purchased from Abcam (ab8580). Reads were aligned to the human genome (hg19, GRCh37) using bwa (version 0.7.12) (Li and Durbin 2009). Only reads that aligned uniquely to a single genomic location $(\mathrm{MAPQ}>10)$ were used for downstream analysis. ChIP-seq peaks and normalized bedGraph files were generated using HOMER using a false discovery rate (FDR) of $0.1 \%$ and fold enrichment over input of at least fourfold (Heinz et al. 2010).

Identification of DamID-seq (DamID combined with next-generation sequencing) peaks

Reads were aligned to the human genome (hg19, GRCh37) using Bowtie2 (version 2.2.3) (Langmead and Salzberg 2012). Only reads that aligned uniquely to a single genomic location were used for downstream analysis. In addition, only reads aligning just downstream from a GATC sequence in the genome were kept for further analysis. Reads aligning to non-GATC locations were much more likely to represent nonspecific noise. Putative DamID-seq peaks were identified using HOMER in a manner similar to variable size ChIP-seq peaks, with several modifications as described previously (Jacinto et al. 2015). Duplicate reads, which are normally discarded to avoid artifacts in ChIP-seq, were retained for DamID-seq analysis, since most of the reads aligned to a limited number of GATC sites. DamID-seq peak regions were required to have twofold more normalized reads than GFP DamID-seq controls. In addition, peaks were required to contain at least 50 normalized reads per peak (per 10 million reads sequenced) to remove low-magnitude sites. Normalized bedGraph files were created for DamID by extending reads $1 \mathrm{~kb}$ both upstream and downstream to reflect the relative size of DamID-enriched regions. Annotation and comparisons between ChIP-seq peaks, DamID-seq peaks, and other genomic features such as the transcriptional start site were performed using the "mergePeaks" and "annotatePeaks.pl" programs in HOMER.

\section{Inducing Nup133 intranuclear localization in C2C12 cells}

Mouse C2C12 cells were split onto coverslips at 30\%-50\% density $24 \mathrm{~h}$ prior to transfection. On the following day, cells were transfected with GFP-Nup98, sPom121-Flag, or both plasmids. After $24 \mathrm{~h}$, cells were fixed in $2 \%$ PFA for $5 \mathrm{~min}$. IF experiments were conducted as discussed above in the "IF and Antibodies" section. Nup133 intranuclear localization intensity was quantified using ImageJ software. GFP-Nup98 foci were circled in the green channel. Next, we switched to the red channel and recorded pixel intensity of Nup133 localization. The pixel intensity of Nup133 foci was compared with neighboring cells that did not overexpress GFP-Nup98 or sPom121-Flag. Untransfected cells did not display any obvious Nup133 intranuclear staining and thus were used as a background measurement. Plots of intranuclear fluorescence were constructed using Prism software.

\section{Acknowledgments}

We thank members of the Hetzer laboratory for helpful comments on the manuscript. We thank the laboratory of Reuben Shaw for use of supplies and expertise. We thank Maureen Powers and Volker Cordes for HeLa-C cells. We thank Bas Van Steensel for constructs that served as templates for subcloning of the DamID vectors used in this study. T.M.F. was supported by American Cancer Society grant PF-11-142-01-DMC. M.W.H. was supported by National Institutes of Health grant R01GM098749, the Glenn Aging Foundation, and American Cancer Society award number P30CA014195. J.M.Y. was supported by a National Institute of Health P50 grant (P50 GM107632; PI: Jef Boeke, New York University). H.S.M. is an Investigator of the Howard Hughes Medical Institute. This work was also partially supported by funds from the Helmsley Charitable Fund, the Mathers Foundation, and the JPB Foundation (F.H.G.).

\section{References}

Alber F, Dokudovskaya S, Veenhoff LM, Zhang W, Kipper J, Devos D, Suprapto A, Karni-Schmidt O, Williams R, Chait BT, et al. 2007. The molecular architecture of the nuclear pore complex. Nature 450: 695-701. 
Antonell A, de Luis O, Domingo-Roura X, Perez-Jurado LA. 2005. Evolutionary mechanisms shaping the genomic structure of the Williams-Beuren syndrome chromosomal region at human 7q11.23. Genome Res 15: 1179-1188.

Antonin W, Franz C, Haselmann U, Antony C, Mattaj IW. 2005. The integral membrane nucleoporin pom121 functionally links nuclear pore complex assembly and nuclear envelope formation. Mol Cell 17: 83-92.

Brawand D, Soumillon M, Necsulea A, Julien P, Csardi G, Harrigan P, Weier M, Liechti A, Aximu-Petri A, Kircher M, et al. 2011. The evolution of gene expression levels in mammalian organs. Nature 478: 343-348.

Brickner DG, Brickner JH. 2011. Gene positioning is regulated by phosphorylation of the nuclear pore complex by Cdk1. Cell Cycle 10: 392-395.

Brickner JH, Walter P. 2004. Gene recruitment of the activated INO1 locus to the nuclear membrane. PLOS Biol 2: e342.

Brohawn SG, Leksa NC, Spear ED, Rajashankar KR, Schwartz TU. 2008. Structural evidence for common ancestry of the nuclear pore complex and vesicle coats. Science 322: 1369-1373.

Buchwalter AL, Liang Y, Hetzer MW. 2014. Nup50 is required for cell differentiation and exhibits transcription-dependent dynamics. Mol Biol Cell 25: 2472-2484.

Burns LT, Wente SR. 2014. From hypothesis to mechanism: uncovering nuclear pore complex links to gene expression. Mol Cell Biol 34: 2114-2120.

Cabal GG, Genovesio A, Rodriguez-Navarro S, Zimmer C, Gadal O, Lesne A, Buc H, Feuerbach-Fournier F, Olivo-Marin JC, Hurt EC, et al. 2006. SAGA interacting factors confine sub-diffusion of transcribed genes to the nuclear envelope. Nature 441: 770-773.

Capelson M, Liang Y, Schulte R, Mair W, Wagner U, Hetzer MW. 2010. Chromatin-bound nuclear pore components regulate gene expression in higher eukaryotes. Cell 140: 372-383.

Casolari JM, Brown CR, Komili S, West J, Hieronymus H, Silver PA. 2004. Genome-wide localization of the nuclear transport machinery couples transcriptional status and nuclear organization. Cell 117: 427-439.

Cortez D, Marin R, Toledo-Flores D, Froidevaux L, Liechti A, Waters PD, Grutzner F, Kaessmann H. 2014. Origins and functional evolution of $\mathrm{Y}$ chromosomes across mammals. Nature 508: 488-493.

DeGrasse JA, DuBois KN, Devos D, Siegel TN, Sali A, Field MC, Rout MP, Chait BT. 2009. Evidence for a shared nuclear pore complex architecture that is conserved from the last common eukaryotic ancestor. Mol Cell Proteomics 8: 2119-2130.

Dennis G Jr, Sherman BT, Hosack DA, Yang J, Gao W, Lane HC, Lempicki RA. 2003. DAVID: Database for Annotation, Visualization, and Integrated Discovery. Genome Biol 4: P3.

Devos D, Dokudovskaya S, Alber F, Williams R, Chait BT, Sali A, Rout MP. 2004. Components of coated vesicles and nuclear pore complexes share a common molecular architecture. PLoS Biol 2: e380.

Devos D, Dokudovskaya S, Williams R, Alber F, Eswar N, Chait BT, Rout MP, Sali A. 2006. Simple fold composition and modular architecture of the nuclear pore complex. Proc Natl Acad Sci 103: 2172-2177.

Dobin A, Davis CA, Schlesinger F, Drenkow J, Zaleski C, Jha S, Batut P, Chaisson M, Gingeras TR. 2013. STAR: ultrafast universal RNA-seq aligner. Bioinformatics 29: 15-21.

Doucet CM, Talamas JA, Hetzer MW. 2010. Cell cycle-dependent differences in nuclear pore complex assembly in metazoa. Cell 141: 1030-1041.
Dultz E, Ellenberg J. 2010. Live imaging of single nuclear pores reveals unique assembly kinetics and mechanism in interphase. J Cell Biol 191: 15-22.

Field MC, Koreny L, Rout MP. 2014. Enriching the pore: splendid complexity from humble origins. Traffic 15: 141-156.

Funakoshi T, Maeshima K, Yahata K, Sugano S, Imamoto F, Imamoto N. 2007. Two distinct human POM121 genes: requirement for the formation of nuclear pore complexes. FEBS Lett 581: 4910-4916.

Funakoshi T, Clever M, Watanabe A, Imamoto N. 2011. Localization of Pom 121 to the inner nuclear membrane is required for an early step of interphase nuclear pore complex assembly. Mol Biol Cell 22: 1058-1069.

Fushan AA, Turanov AA, Lee SG, Kim EB, Lobanov AV, Yim SH, Buffenstein R, Lee SR, Chang KT, Rhee H, et al. 2015. Gene expression defines natural changes in mammalian lifespan. Aging Cell 14: 352-365.

Griffis ER, Altan N, Lippincott-Schwartz J, Powers MA. 2002. Nup98 is a mobile nucleoporin with transcription-dependent dynamics. Mol Biol Cell 13: 1282-1297.

Griffis ER, Craige B, Dimaano C, Ullman KS, Powers MA. 2004. Distinct functional domains within nucleoporins Nup153 and Nup98 mediate transcription-dependent mobility. Mol Biol Cell 15: 1991-2002.

Guillaume F, Otto SP. 2012. Gene functional trade-offs and the evolution of pleiotropy. Genetics 192: 1389-1409.

Heinz S, Benner C, Spann N, Bertolino E, Lin YC, Laslo P, Cheng JX, Murre C, Singh H, Glass CK. 2010. Simple combinations of lineage-determining transcription factors prime cis-regulatory elements required for macrophage and B cell identities. Mol Cell 38: 576-589.

Hoelz A, Debler EW, Blobel G. 2011. The structure of the nuclear pore complex. Annu Rev Biochem 80: 613-643.

Hurt E, Beck M. 2015. Towards understanding nuclear pore complex architecture and dynamics in the age of integrative structural analysis. Curr Opin Cell Biol 34: 31-38.

Ibarra A, Hetzer MW. 2015. Nuclear pore proteins and the control of genome functions. Genes Dev 29: 337-349.

Jacinto FV, Benner C, Hetzer MW. 2015. The nucleoporin Nup153 regulates embryonic stem cell pluripotency through gene silencing. Genes Dev 29: 1224-1238.

Jacob F. 1977. Evolution and tinkering. Science 196: 1161-1166.

Kalverda B, Fornerod M. 2010. Characterization of genome-nucleoporin interactions in Drosophila links chromatin insulators to the nuclear pore complex. Cell Cycle 9: 4812-4817.

Kasper LH, Brindle PK, Schnabel CA, Pritchard CE, Cleary ML, van Deursen JM. 1999. CREB binding protein interacts with nucleoporin-specific FG repeats that activate transcription and mediate NUP98-HOXA9 oncogenicity. Mol Cell Biol 19: $764-776$.

Kipersztok S, Osawa GA, Liang LF, Modi WS, Dean J. 1995. POMZP3, a bipartite transcript derived from human ZP3 and a POM121 homologue. Genomics 25: 354-359.

Langmead B, Salzberg SL. 2012. Fast gapped-read alignment with Bowtie 2. Nat Methods 9: 357-359.

Leksa NC, Schwartz TU. 2010. Membrane-coating lattice scaffolds in the nuclear pore and vesicle coats: commonalities, differences, challenges. Nucleus 1: 314-318.

Li H, Durbin R. 2009. Fast and accurate short read alignment with Burrows-Wheeler transform. Bioinformatics 25: 1754-1760.

Liang Y, Franks TM, Marchetto MC, Gage FH, Hetzer MW. 2013. Dynamic association of NUP98 with the human genome. PLoS Genet 9: e1003308.

Light WH, Freaney J, Sood V, Thompson A, D'Urso A, Horvath CM, Brickner JH. 2013. A conserved role for human Nup98 
in altering chromatin structure and promoting epigenetic transcriptional memory. PLOS Biol 11: e1001524.

Mitchell JM, Mansfeld J, Capitanio J, Kutay U, Wozniak RW. 2010. Pom 121 links two essential subcomplexes of the nuclear pore complex core to the membrane. I Cell Biol 191: 505-521.

Morchoisne-Bolhy S, Geoffroy MC, Bouhlel IB, Alves A, Auduge N, Baudin X, Van Bortle K, Powers MA, Doye V. 2015. Intranuclear dynamics of the Nup107-160 complex. Mol Biol Cell 26: 2343-2356.

Neumann N, Lundin D, Poole AM. 2010. Comparative genomic evidence for a complete nuclear pore complex in the last eukaryotic common ancestor. PLoS One 5: e13241.

Orr HA. 2000. Adaptation and the cost of complexity. Evolution 54: $13-20$.

O'Sullivan JM, Tan-Wong SM, Morillon A, Lee B, Coles J, Mellor J, Proudfoot NJ. 2004. Gene loops juxtapose promoters and terminators in yeast. Nat Genet 36: 1014-1018.

Pascual-Garcia P, Jeong J, Capelson M. 2014. Nucleoporin Nup98 associates with Trx/MLL and NSL histone-modifying complexes and regulates Hox gene expression. Cell Rep 9: 433-442.

Ptak C, Aitchison JD, Wozniak RW. 2014. The multifunctional nuclear pore complex: a platform for controlling gene expression. Curr Opin Cell Biol 28: 46-53.

Rabut G, Doye V, Ellenberg J. 2004. Mapping the dynamic organization of the nuclear pore complex inside single living cells. Nat Cell Biol 6: 1114-1121.

Raices M, D'Angelo MA. 2012. Nuclear pore complex composition: a new regulator of tissue-specific and developmental functions. Nat Rev Mol Cell Biol 13: 687-699.
Robinson MD, McCarthy DJ, Smyth GK. 2010. edgeR: a Bioconductor package for differential expression analysis of digital gene expression data. Bioinformatics 26: 139-140.

Shaulov L, Gruber R, Cohen I, Harel A. 2011. A dominant-negative form of POM121 binds chromatin and disrupts the two separate modes of nuclear pore assembly. I Cell Sci 124: 3822-3834.

Solmaz SR, Chauhan R, Blobel G, Melcak I. 2011. Molecular architecture of the transport channel of the nuclear pore complex. Cell 147: 590-602.

Taddei A, Van Houwe G, Hediger F, Kalck V, Cubizolles F, Schober H, Gasser SM. 2006. Nuclear pore association confers optimal expression levels for an inducible yeast gene. Nature 441: 774-778.

Talamas JA, Hetzer MW. 2011. POM121 and Sun1 play a role in early steps of interphase NPC assembly. J Cell Biol 194: 27-37.

Tan-Wong SM, Wijayatilake HD, Proudfoot NJ. 2009. Gene loops function to maintain transcriptional memory through interaction with the nuclear pore complex. Genes Dev 23: 2610-2624.

van Steensel B, Henikoff S. 2000. Identification of in vivo DNA targets of chromatin proteins using tethered dam methyltransferase. Nat Biotechnol 18: 424-428.

Wente SR, Rout MP. 2010. The nuclear pore complex and nuclear transport. Cold Spring Harb Perspect Biol 2: a000562.

Xu S, Powers MA. 2010. Nup98-homeodomain fusions interact with endogenous Nup98 during interphase and localize to kinetochores and chromosome arms during mitosis. Mol Biol Cell 21: 1585-1596.

Yavuz S, Santarella-Mellwig R, Koch B, Jaedicke A, Mattaj IW, Antonin W. 2010. NLS-mediated NPC functions of the nucleoporin Pom121. FEBS Lett 584: 3292-3298. 


\section{CORRIGENDUM}

Genes \& Development 30: 1155-1171 (2016)

\section{Corrigendum: Evolution of a transcriptional regulator from a transmembrane nucleoporin}

Tobias M. Franks, Chris Benner, Iñigo Narvaiza, Maria C.N. Marchetto, Janet M. Young, Harmit S. Malik, Fred H. Gage, and Martin W. Hetzer

In the version of this article initially published, the funding source R01 NS096786 was missing from the acknowledgments.

doi: $10.1101 /$ gad.300699.117 


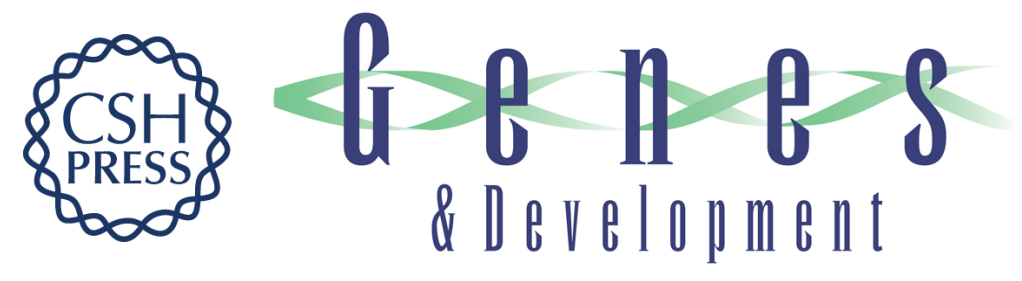

\section{Evolution of a transcriptional regulator from a transmembrane nucleoporin}

Tobias M. Franks, Chris Benner, Iñigo Narvaiza, et al.

Genes Dev. 2016, 30: originally published online May 19, 2016

Access the most recent version at doi:10.1101/gad.280941.116

\section{Supplemental http://genesdev.cshlp.org/content/suppl/2016/05/19/gad.280941.116.DC1 \\ Material}

Related Content

Corrigendum: Evolution of a transcriptional regulator from a transmembrane nucleoporin

Tobias M. Franks, Chris Benner, Iñigo Narvaiza, et al.

Genes Dev. April , 2017 31: 845

References This article cites 60 articles, 22 of which can be accessed free at: http://genesdev.cshlp.org/content/30/10/1155.full.html\#ref-list-1

Articles cited in: http://genesdev.cshlp.org/content/30/10/1155.full.html\#related-urls

Creative This article, published in Genes \& Development, is available under a Creative Commons Commons License (Attribution 4.0 International), as described at License http://creativecommons.org/licenses/by/4.0/.

Email Alerting Receive free email alerts when new articles cite this article - sign up in the box at the top Service right corner of the article or click here.

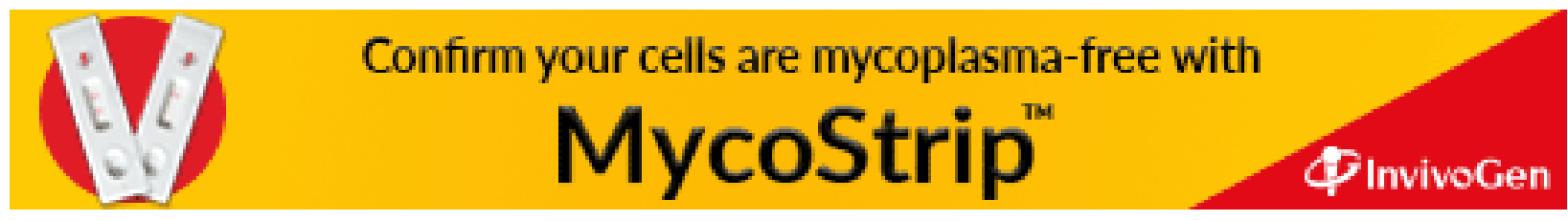

